\title{
Electrical and Noise Modeling of GaAs Schottky Diode Mixers in the THz Band
}

\author{
Diego Pardo, Jesús Grajal, and Susana Pérez
}

\begin{abstract}
This paper presents a simulation tool for the analysis and design of Schottky mixers which is able to evaluate self-consistently both the conversion losses and the noise temperature. The tool is based on a Monte Carlo model of the diode coupled with a multi-tone harmonic balance technique. A remarkable feature of this tool is that it avoids the need of analytical or empirical models. The validation of the tool has been carried out by comparing simulation results with measurements of mixers published in the literature up to $2.5 \mathrm{THz}$. The usefulness of Schottky diodes as frequency mixers above $2.5 \mathrm{THz}$ is analyzed. Additionally, the range of application and the limitations of simpler simulation tools based on lumped equivalent circuits or drift-diffusion models have been evaluated using the Monte Carlo model as a reference.
\end{abstract}

Index Terms-Drift-diffusion, frequency mixer, harmonic balance technique, lumped equivalent circuit (LEC), Monte Carlo, numerical modeling, Schottky diode, $\mathrm{THz}$ frequencies.

\section{INTRODUCTION}

$\mathbf{T}$ HE development of sources and detectors at millimeter and submillimeter wavelengths has been mainly driven by applications in astronomy and space science [1]-[3]. In recent years, with terahertz technology becoming mature, new applications appear in different topics such as Earth observation, medical imaging, security, radar and communications, among others [1], [4].

GaAs Schottky diode technology has been widely used at millimeter and submillimeter wavelengths in the last decades due to the excellent trade-off between mobility and bandgap in GaAs, and also to the simplicity and maturity of GaAs processing [1]. The use of Schottky technology for receivers is getting popular for application such as Earth observation, planetary science and ground applications (medicine, security, etc.) [1], [4], [5]. Schottky mixers offer good enough sensitivities and performance for these applications, and do not need to be cooled,

Manuscript received February 17, 2015; revised July 07, 2015 and October 07, 2015; accepted November 05, 2015. Date of publication December 07, 2015; date of current version January 20, 2016. This work was supported by the European Commissionunder Project MIDAS FP7-SPACE-2009-1 242334), by the Spanish National Research and Development Program under Projects TEC2011-28683-C02-01, TEC2013-41640-R, TEC2014-53815-R, and TeraSense (Consolider-Ingenio 2010, CDS2008-00068), by the Madrid Regional Governmentunder project S2013/ICE-3000 (SPADERADAR-CM) and by the Junta de Castilla y Leónunder project SA052U13.

D. Pardo and J. Grajal are with the Signal, System and Radiocommunications Department, Universidad Politécnica de Madrid, E.T.S.I. Telecomunicación, Av. Complutense 30, 28040 Madrid, Spain (e-mail: dpardo@gmr.ssr.upm.es; jesus@gmr.ssr.upm.es).

S. Pérez is with the Applied Physics Department, Universidad de Salamanca, Plaza de la Merced s/n, 37008 Salamanca, Spain, (e-mail: susana@usal.es).

Color versions of one or more of the figures in this paper are available online at http://ieeexplore.iee.org.

Digital Object Identifier 10.1109/TTHZ.2015.2502061 contrary to HEB and SIS mixers, with the subsequent reduction in system sizes and costs.

In order to effectively design Schottky mixers, both the electrical and noise performances of the devices have to be analyzed in a fully self-consistent manner. Most of the simulation tools used in the design of Schottky mixers are based on the conversion matrix (CM) formalism [6] where the diode is modeled analytically by a lumped equivalent circuit (LEC). LEC models do not take into account important physical effects within the semiconductor device, such as velocity saturation and nonlocal effects in time and space [7]-[13]. Hence, the use of LEC models has limited validity at high frequencies and power levels. As regards noise sources, simulation tools based on the CM usually take into account shot and thermal noise as well as the effects of the modulation of the noise sources by the LO excitation. However they present important deficiencies to describe the excess noise due to hot electrons and intervalley transfer that appears in the device under high field conditions [14], [15]. Circuit simulators based on physics-based models like drift-diffusion or hydrodynamic models have been developed, where noise can be evaluated with the transfer-impedance field method [16]-[19]. However, a fundamental limitation of these techniques is that they require to know the spectral performance of the noise sources, usually provided by Monte Carlo simulations.

The scope of this paper is to present a simulation tool which enables the analysis and design of Schottky mixers from both electrical and noise considerations, without the necessity of any additional analytical or empirical model. This tool is based on the multi-tone harmonic balance (HB) technique together with the Monte Carlo (MC) model. The MC model [20], [21] provides a unified and self-consistent description of the electrical and the noise performance of the device [22]-[24]. The validation of our simulation tool has been carried out by comparing simulations and measured results available in the literature. Additionally, this simulation tool has been used to analyze both the electrical and noise responses of Schottky mixers at $\mathrm{THz}$ frequencies and the physical effects that limit the performance of these circuits. Also the range of validity and the limitations of simpler simulation tools based on lumped equivalent circuits or drift-diffusion models have been evaluated using the Monte Carlo model as a reference.

The description of the simulation tool and the procedure for the evaluation of the mixer noise are described in Section II. The validation of the CAD tool is outlined in Section III, including a comparison of measured and simulated conversion losses and noise temperature for fundamental and subharmonically-pumped (SHP) mixers. An analysis of the performance of frequency mixers above $2.5 \mathrm{THz}$ is presented in Section IV. In 


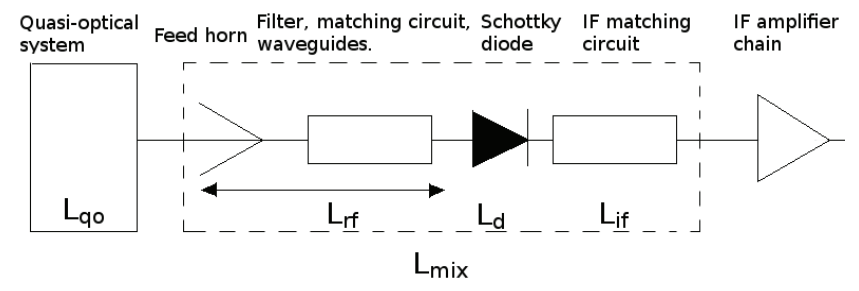

Fig. 1. Block diagram of a Schottky receiver.

Section $\mathrm{V}$, the predictions of our simulation tool are compared with other CAD tools based on drift-diffusion and lumped equivalent circuit models. Some conclusions are drawn in Section VI.

\section{SimULATION FRAMEWORK}

This section presents a brief description of the technique for modeling Schottky-based mixers using the MC model [25].

\section{A. Figures of Merit of the Receiver}

The block diagram of a Schottky diode receiver is shown in Fig. 1. The receiver consists of different elements which contribute to the total losses and noise temperature of the system. The total losses of the receiver are composed of $L_{\mathrm{qo}}$, losses in the quasi-optical parts of the systems (lens, diplexers, etc.), $L_{\mathrm{rf}}$, losses in the feed-horn, filters and mixer waveguides, $L_{d}$, the diode conversion losses, and $L_{\mathrm{if}}$, the resistive and mismatch losses in the IF matching circuit [26]-[28]:

$$
L_{\mathrm{rec}}=L_{\mathrm{qo}} L_{\mathrm{rf}} L_{d} L_{\mathrm{if}} .
$$

The equivalent input noise temperature of the receiver is evaluated using Friis' formula:

$T_{\mathrm{rec}, \mathrm{DSB}}=\left(L_{\mathrm{qo}}-1\right) T_{\mathrm{phys}}+L_{\mathrm{qo}} T_{\mathrm{mix}, \mathrm{DSB}}+L_{\mathrm{qo}} L_{\mathrm{mix}, \mathrm{DSB}} T_{\mathrm{if}}$

where DSB means double sideband ${ }^{1}, T_{\text {phys }}$ is the physical temperature and $T_{\mathrm{if}}$ is the noise temperature of the IF amplifier chain. This formula assumes that the different stages of the receiver are matched [29]. The mixer conversion loss $L_{\mathrm{mix}, \mathrm{DSB}}$ and noise temperature $T_{\mathrm{mix}, \mathrm{DSB}}$ are defined as:

$$
\begin{aligned}
L_{\text {mix }, \mathrm{DSB}}= & L_{\mathrm{rf}} L_{d, \mathrm{DSB}} L_{\mathrm{if}} \\
T_{\text {mix }, \mathrm{DSB}}= & \left(L_{\mathrm{rf}}-1\right) T_{\mathrm{phys}}+L_{\mathrm{rf}} T_{d, \mathrm{DSB}} \\
& +L_{\mathrm{rf}} L_{d, \mathrm{DSB}}\left(L_{\mathrm{if}}-1\right) T_{\mathrm{phys}} .
\end{aligned}
$$

Our simulator calculates the diode conversion losses $L_{d, \mathrm{SSB}}$ and noise temperature $T_{d, \mathrm{SSB}}$ and they are transformed into the corresponding DSB mixer or receiver ones using these equations and the data provided in the literature for $L_{\mathrm{qo}}, L_{\mathrm{rf}}, L_{\mathrm{if}}$ and $T_{\mathrm{if}}$.

Three different types of mixers have been considered in this work: Fundamental single-ended (F-SE) mixer with a single Schottky diode, fundamental balanced (F-B) mixers based on two diodes in a balanced configuration, and subharmonically pumped (SHP) mixers based on an antiparallel diode pair. The

\footnotetext{
${ }^{1}$ It has been assumed that the single sideband and double sideband conversion loss and noise temperature are related by [29]: $L_{d, \mathrm{SSB}}(\mathrm{dB})=L_{d, \mathrm{DSB}}(\mathrm{dB})+$ $3 \mathrm{~dB}$ and $T_{d, \mathrm{SSB}}(K)=2 \cdot T_{d, \mathrm{DSB}}(K)$.
}

simulation of the single-ended fundamental mixer is straightforward. To simulate the balance fundamental mixers, an equivalent single-diode mixer having the same conversion losses and noise temperature has been used [29]. For SHP mixers, the simulator analyses the real structure with two diodes in an antiparallel connection.

\section{B. Description of the Monte Carlo Model}

The Monte Carlo model provides a solution for the Boltzmann transport equation by simulating the trajectories of individual carriers as they move through a device under the influence of electric fields and random scattering forces [20], [21], [30]. Therefore, this technique provides an accurate description of physical phenomena in the device up to THz frequencies. An ensemble Monte Carlo self-consistently coupled with a one-dimensional Poisson solver has been used in this paper [24], [31]. The ohmic contact is modeled as a surface that injects carriers in thermal equilibrium with the lattice. In addition, any carrier reaching the contact leaves the device. On the other hand, the Schottky contact is simulated as a perfect absorbent surface. Scattering mechanisms included in the Monte Carlo simulation are ionized impurities, acoustic phonon, polar and non-polar optical phonon, and intervalley mechanisms. The band structure is modeled as a conduction band with three spherical non-parabolic valleys [20].

The MC method is recognized as the most rigorous technique for noise simulation since it allows the appropriate correlation functions to be calculated directly from a multi-particle history simulated during a sufficiently long-time interval [24]. Shot noise, thermal noise, hot electron noise and intervalley transfer noise [14], [22], [24], [31]-[33] as well as the effects due to the modulation of the noise sources by the voltage waveforms are inherently taken into account with this method. Besides, the coupling of fluctuations of the electron velocity and the electric field is also simulated with the MC model. The MC model has been successfully used to describe noise features of GaAs Schottky diodes under static and large-signal conditions [22]-[24], [31], [33].

In order to obtain reliable results for the conversion losses calculated with the MC model, the number of simulated particles has to be chosen large enough. Additionally, to calculate the noise temperature, the number of simulated particles and also the duration of the simulated history have to be sufficiently large to accurately evaluate the statistics of the current/voltage fluctuations of the device. Fig. 2 shows the conversion losses of the 585-690 GHz fundamental mixer in [34] simulated with the Monte Carlo model coupled to the harmonic balance technique (MCHB) as a function of the RF power $\left(P_{\mathrm{RF}}\right)$ and the number of simulated particles. As the $P_{\mathrm{RF}}$ considered in the simulations decreases, the minimum number of simulated particles to assure accurate results with the $\mathrm{MC}$ model increases. In the simulations presented in this work, $P_{\mathrm{RF}}=-20 \mathrm{dBm}$ and more than 10000 particles in the MC model have been considered.

\section{Coupling a Physical Device Model With a Circuit Simulator for Mixer Analysis}

The HB method is the most common technique for the design of large-signal nonlinear microwave circuits [35]-[37]. The integration of numerical simulators for active devices into circuit 


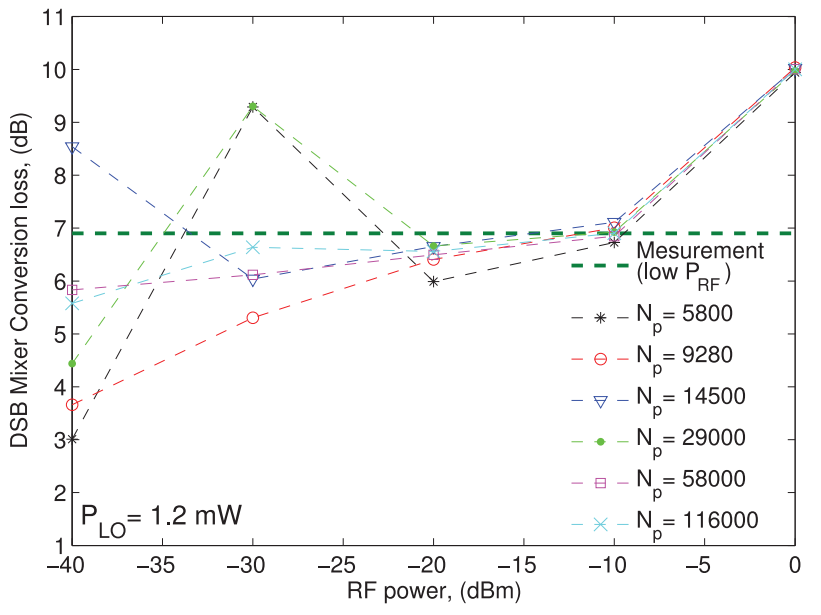

Fig. 2. Conversion losses of the $585 \mathrm{GHz}-690 \mathrm{GHz}$ fundamental mixer in [34] obtained with MCHB as a function of the number of simulated particles for different values of RF power. The LO power has been fixed to $1.2 \mathrm{~mW}$, where the diode is operating in the vicinity of flat band, see Section III.

simulators avoids the need of an equivalent circuit model extraction. With this technique, the device-circuit interaction is taken into account and the mixers can be designed from both a device and circuit point-of-view [13].

The HB technique incorporates the benefits of the frequency domain analysis for the linear part of the circuit and the time domain analysis for the nonlinear part of the circuit. The time-domain current in the diode is evaluated using the $\mathrm{MC}$ model under the voltage waveform provided by the HB technique. This current is converted to the frequency domain, and then, different algorithms can be used to update the voltage applied to the terminals of the diode in terms of the circuit embedding impedances, the harmonic components of the current and the harmonic components of the voltage from the previous iterations [13], [37]. In mixers, the presence of multi-tone excitations makes necessary to take into account not only the different harmonics of the LO and the RF signals but also their intermodulation products $m f_{\mathrm{LO}}+n f_{\mathrm{RF}}$, with $m$ and $n$ integers. When $f_{\mathrm{LO}}$ and $f_{\mathrm{RF}}$ are commensurate, i.e., the quotient $f_{\mathrm{LO}} / f_{\mathrm{RF}}$ is rational, the multi-tone excitation can be defined as a periodic waveform by using the greatest common divisor of the LO and the RF frequencies, so the fast Fourier transform (FFT) can be employed. In the case where $f_{\mathrm{LO}}$ and $f_{\mathrm{RF}}$ are non-commensurate, the currents and voltages are not periodic, and thus the FFT is not applicable. There are several algorithms which can be used in this case [35]-[38]. Our multi-tone HB is based on the almost periodic Fourier transform (APTF) which is applicable to physics-based numerical models with memory [38].

A $5 \times 3$ box truncation scheme has been considered in simulations, where 5 and 3 are the highest harmonics of the LO and the RF frequencies, respectively (the total number of frequency components used with this scheme is $39, f_{m, n}=m f_{\mathrm{LO}}+n f_{\mathrm{RF}}$ where $|m| \leq 5,|n| \leq 3$ and $\left.f_{m, n} \geq 0\right)$. Due to the practical impossibility of achieving optimum terminations at a large number of mixing frequencies, the impedances at mixing frequencies other than LO, RF, IF, and image frequencies have been set to zero in the simulations. Our simulator provides the possibility to conjugately match automatically the input impedances at the LO and RF frequencies at each operating point.

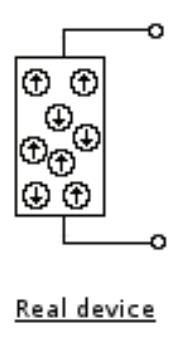

(a)

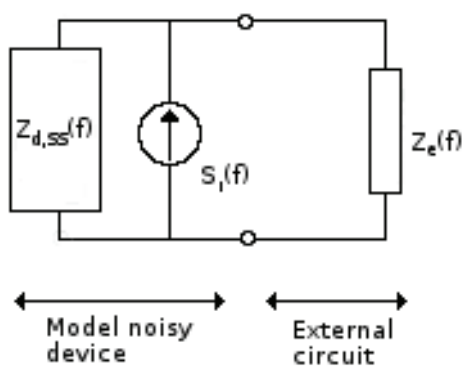

(b)
Fig. 3. (a) Noisy device and (b) equivalent circuit of a mixer at frequency $f$.

The presence of parasitic elements in the circuits, which may result in a degradation of the mixer performance, can be taken into account with our simulator. These elements - usually a parasitic capacitance and a parasitic inductance-are absorbed in the linear part of the circuit, see [13] for further details.

\section{Evaluation of the Equivalent Input Noise Temperature of the Mixers With the MCHB Tool}

Using Norton's theorem, a noisy diode can be represented by a noise current generator characterized by a current noise spectral density $S_{I}(f)$ obtained under short-circuit conditions in parallel with the small-signal impedance of the diode $Z_{d, \mathrm{SS}}$ or by a voltage noise generator characterized by a voltage noise spectral density $S_{V}(f)$ obtained under open-circuit conditions in series with $Z_{d, \mathrm{SS}}$ [39], see Fig. 3 . The impedance $Z_{d, \mathrm{SS}}$ is a small-signal impedance which relates the fluctuations of the current and the voltage at the terminals the diode

$$
Z_{d, \mathrm{SS}}(f)=\left.\frac{V_{\mathrm{SS}}(f)}{I_{\mathrm{SS}}(f)}\right|_{V_{\mathrm{LO}}}
$$

where $V_{\mathrm{LO}}$ indicates that the diode is driven by the LO excitation, $V_{\mathrm{SS}}(f)$ is a small-signal sinusoidal voltage of frequency $f$ superimposed to the LO signal at the terminals of the diode and $I_{\mathrm{SS}}(f)$ is the coefficient of the Fourier series of the small-signal current of frequency $f$ (usually $f$ is the intermediate frequency $\left.f_{\mathrm{IF}}\right)$.

In this section, a description of $S_{I}(f)$ and $Z_{d, \mathrm{SS}}$ is presented as well as their evaluation with the MCHB tool in order to calculate the noise temperature of the mixers.

1) Calculation of $S_{I}(f)$ : Firstly, the MCHB tools is used to calculate the voltage waveforms at the terminals of the diode and the conversion losses of the mixer for a given LO power. Then, the Schottky diode is simulated with the MC model under the voltage waveforms obtained with the MCHB tool, and the current noise spectral density $S_{I}(f)$ is evaluated. The current fluctuations $\delta I(t)^{2}$ obtained from the MC simulations can be used to calculate the correlation function $R_{I}(t, \tau)=\langle\delta I(t+$ $\tau / 2) \delta I(t-\tau / 2)\rangle$, where $t \in\left[0, T_{\mathrm{IF}}\right]$ and $T_{\mathrm{IF}}$ is the period of the IF signal. The Fourier transform of $R_{I}(t, \tau)$ with respect to the correlation time $\tau$ determines the spectral density of the current fluctuations at time moment $t, S_{I}(t, f)$. The conventional power spectral density $S_{I}(f)$ used in this work is obtained by averaging $S_{I}(t, f)$ over the time $t$ [24], [40].

${ }^{2} I(t)=\langle I(t)\rangle+\delta I(t)$ where $\langle I(t)\rangle$ is the deterministic part of the current and the brakets $\langle\ldots\rangle$ mean averaging over an ensemble of different realizations of $I(t)$ histories. 
TABLE I

Parameters of Schottky Diode Mixers Published in the Literature and Data Used in Our Simulations

\begin{tabular}{|c|c|c|c|c|c|c|c|c|c|c|c|c|c|c|c|c|c|c|c|}
\hline \multicolumn{20}{|c|}{ Data published in the literature for Schottky mixers } \\
\hline Mixer, $(\mathrm{GHz})$ & $\begin{array}{c}f_{R F}, \\
(\mathrm{GHz})\end{array}$ & \begin{tabular}{|c|}
$f_{L O}$, \\
$(\mathrm{GHz})$
\end{tabular} & $\begin{array}{l}P_{L O}, \\
(\mathrm{~mW})\end{array}$ & $\begin{array}{c}\mathrm{V}_{\text {bias }}, \\
\text { (V) }\end{array}$ & $\begin{array}{c}\mathrm{N}_{e} \\
\left(\mathrm{~cm}^{-3}\right)\end{array}$ & $\begin{array}{l}\mathrm{L}_{e}, \\
\mathrm{~nm})\end{array}$ & $\begin{array}{l}\text { Area, } \\
\left(\mu m^{2}\right)\end{array}$ & $\begin{array}{l}\phi_{b}, \\
(\mathrm{~V})\end{array}$ & \begin{tabular}{l|}
$\mathrm{R}_{s}{ }^{\mathbf{f}}$ \\
$(\Omega)$
\end{tabular} & $\begin{array}{c}\mathrm{C}_{j}(0) \\
(\mathrm{fF})\end{array}$ & $\begin{array}{c}Z_{R F} \\
(\Omega)\end{array}$ & $\begin{array}{c}Z_{L O} \\
(\Omega)\end{array}$ & $\begin{array}{r}Z_{I F} \\
(\Omega)\end{array}$ & $\begin{array}{c}\mathrm{L}_{q o} \\
(\mathrm{~dB})\end{array}$ & $\begin{array}{l}\mathrm{L}_{r f} \\
(\mathrm{~dB})\end{array}$ & $\begin{array}{l}\mathrm{L}_{i f} \\
(\mathrm{~dB})\end{array}$ & \begin{tabular}{|c|}
$\mathrm{L}_{\text {mix }}$, \\
$(\mathrm{dB}, \mathrm{DSB})$
\end{tabular} & $\begin{array}{c}\mathrm{T}_{\text {mix }} \\
(\mathrm{K}, \mathrm{DSB})\end{array}$ & \begin{tabular}{|l|}
$\mathbf{T}_{i f}$ \\
$(\mathrm{~K})$
\end{tabular} \\
\hline 86-106 F-SE [27], [46] & 93.4 & 92 & $1.5-2$ & 0.65 & $2 \times 10^{17}$ & 100 & 4.91 & 0.85 & 7 & 6 & $80-\mathrm{j} 1$ & $64+\mathrm{j} 51 \quad 1$ & $110+\mathrm{j} 8^{\mathrm{b}}$ & & 1.2 & & 5.5 & 770 & 129 \\
\hline $300-360$ SHP [28], [47] & 330 & 164.251 & $1.5-5.5$ & 0 & $2 \times 10^{17}$ & 100 & 0.8 & 0.85 & $11-15$ & 1.3 & $83+\mathrm{j} 53$ & $147+\mathrm{j} 207$ & 100 & & 0.7 & 1 & 6 & 700 & \\
\hline 585-690 F-SE [34], [42] & 585 & 583.2 & 1.16 & & $2 \times 10^{17}$ & & 1.13 & & 14 & 2 & $45+j 30$ & $45+j 30$ & 150 & 0.7 & 1.6 & 0.7 & 6.9 & 1490 & 100 \\
\hline $835-900$ F-B [48], [49] & 865.8 & & 1 & $\sim 0.65$ & $5 \times 10^{17}$ & & 0.4 & 0.73 & 30 & 1 & $90+\mathrm{j} 200$ & $17+\mathrm{j} 50$ & 200 & & 0.7 & 1.2 & 9.25 & 2660 & \\
\hline 1200 SHP [50], [51] & $\sim 1200$ & $\sim 6001$ & $.5-2.5$ & $\begin{array}{l}50.5 \\
\end{array}$ & & & & & & & & & & & & & $10.5^{\mathrm{h}}$ & $2828^{h}$ & \\
\hline $2500 \mathrm{~F}-\mathrm{SE}[52]$ & 2514 & 2522 & 6 & $0.3-0.6$ & $1 \times 10^{18}$ & 100 & 0.35 & & $15-20$ & & & & & 2 & & 0.4 & 14.9 & 5665 & 150 \\
\hline 4750 F-SE [53] ${ }^{a}$ & 4744 & 4750 & 1 & & $1 \times 10^{18}$ & 30 & 0.05 & & $20-40$ & 0.25 & & & & & & & & 70000 & 30 \\
\hline \multicolumn{20}{|c|}{ Data for Schottky mixers in our simulations } \\
\hline Mixer, $(\mathrm{GHz})$ & $\begin{array}{c}f_{R F}, \\
(\mathrm{GHz})\end{array}$ & \begin{tabular}{|c|}
$f_{L O}$, \\
$(\mathrm{GHz})$
\end{tabular} & \begin{tabular}{|l|}
$P_{L O}$, \\
$(\mathrm{mW})$
\end{tabular} & $\begin{array}{c}\mathrm{V}_{\text {bias }}, \\
\text { (V) }\end{array}$ & $\begin{array}{c}\mathrm{N}_{e} \\
\left(\mathrm{~cm}^{-3}\right)\end{array}$ & $\begin{array}{c}\mathrm{L}_{e}, \\
\mathrm{~nm})\end{array}$ & $\begin{array}{l}\text { Area, } \\
\left(\mu m^{2}\right)\end{array}$ & $\begin{array}{l}\phi_{b}, \\
(\mathrm{~V})\end{array}$ & $\begin{array}{l}\mathrm{R}_{s}, \\
(\Omega)\end{array}$ & $\begin{array}{c}\mathrm{C}_{j}(0) \\
(\mathrm{fF})\end{array}$ & $\begin{array}{c}Z_{R F} \\
(\Omega)\end{array}$ & $\begin{array}{c}Z_{L O} \\
(\Omega)\end{array}$ & $\begin{array}{l}Z_{I F} \\
(\Omega)\end{array}$ & $\begin{array}{l}\mathrm{L}_{q o} \\
(\mathrm{~dB})\end{array}$ & & $\begin{array}{l}\mathrm{L}_{i f} \\
(\mathrm{~dB})\end{array}$ & $\begin{array}{l}\mathrm{L}_{\text {mix }}, \\
(\mathrm{dB}, \mathrm{DSB})\end{array}$ & $\begin{array}{l}\mathrm{T}_{\text {mix }}, \\
(\mathrm{K}, \mathrm{DSB})\end{array}$ & $\begin{array}{l}\mathrm{T}_{i f} \\
(\mathrm{~K})\end{array}$ \\
\hline $86-106$ F-SE [27], [46] ${ }^{\mathrm{g}}$ & 100 & 90 & 1.5 & 0.65 & $2 \times 10^{17}$ & 100 & 4.91 & 0.85 & 7.01 & 7.3 & $80-\mathrm{j} 1$ & $64+\mathrm{j} 51$ & $110+\mathrm{j} 8$ & & 1.2 & & 3.7 & 360 & 120 \\
\hline $300-360$ & 360 & 168 & $1.5-5.5$ & 0 & $2 \times 10^{17}$ & $80^{c}$ & 0.8 & $0.95^{\mathrm{d}}$ & $18.1^{\mathrm{c}}$ & 1.15 & $83+\mathrm{j} 53$ & $147+\mathrm{j} 207$ & 100 & & 0.7 & 1 & 5.7 & 700 & \\
\hline$[34],[42]^{g}$ & 585 & 580 & 1.16 & 0.6 & $2 \times 10^{17}$ & 80 & 1.13 & 0.85 & 15 & 1.8 & $45+\mathrm{j} 30$ & $45+\mathrm{j} 30$ & 150 & 0.7 & 1.6 & 0.7 & 6.8 & 1710 & 100 \\
\hline $835-900$ F-B [48], [49] & 900 & 870 & 1 & 0.65 & $5 \times 10^{17}$ & 100 & 0.4 & 0.75 & 30.3 & 1 & $90+\mathrm{j} 200$ & $17+\mathrm{j} 50$ & 200 & & 0.7 & 1.2 & 9.9 & 2000 & \\
\hline 1200 SHP [50], [51] ${ }^{\mathrm{g}}$ & 1230 & 600 & $1.5-5.5$ & 0.5 & $5 \times 10^{17}$ & 100 & 0.5 & 0.8 & 20 & 1.21 & $27+\mathrm{j} 14$ & $38+\mathrm{j} 33$ & 50 & & 0.7 & 1.2 & 11.5 & 3590 & \\
\hline $2500 \mathrm{~F}-\mathrm{SE}[52]$ & 2500 & 2475 & 6 & 0.6 & $1 \times 10^{18}$ & 100 & 0.35 & 0.99 & 20.27 & 1.07 & $70+\mathrm{j} 113$ & $86+j 36$ & 250 & 2 & & \begin{tabular}{|l|}
0.4 \\
\end{tabular} & $9-13$ & $\sim 5350$ & 150 \\
\hline 4750 F-SE [53] ${ }^{a}$ & 4750 & 4725 & 1 & 0.6 & $1 \times 10^{18}$ & 30 & $0.07^{\mathrm{e}}$ & 0.99 & 40 & 0.25 & $130+\mathrm{j} 220$ & $130+j 220$ & 250 & 2 & & 0.4 & \begin{tabular}{l|l}
$10-33$ & 4 \\
\end{tabular} & $4600-2 \times 10^{5}$ & 30 \\
\hline $\begin{array}{l}\text { a Conversion losses and } \\
\text { the receiver. } \\
\text { b Measured circuit impe } \\
\mathrm{Z}\left[2 f_{L O}-f_{R F}\right]=\mathrm{Z}[ \\
\text { c Physics-based models } \\
\text { to a dc series resistan } \\
\text { epilayer length has be } \\
\text { d } \phi_{b} \text { has been slightly s } \\
\text { e The anode area has be } \\
\text { f } R_{s} \text { given in the literat } \\
\text { g Parasitic capacitances } \\
\text { SHP mixer, } C_{p}=5 \mathrm{fF}\end{array}$ & $\begin{array}{l}\text { noise ter } \\
\text { dances } \\
f_{R F}=(8 \\
\text { with the } \\
\text { e around } \\
\text { en reduce } \\
\text { hifted wi } \\
\text { en select }\end{array}$ & $\begin{array}{l}\text { emperatu } \\
\text { at diffe } \\
80-1 \mathrm{j}) \Omega \\
\text { parame } \\
\text { d } 22 \Omega \\
\text { ed from } \\
\text { ith resp } \\
\text { ted to } r\end{array}$ & $\begin{array}{l}\text { ture are } \\
\text { erent in } \\
\Omega \text {. Thes } \\
\text { heters gi } \\
2 \text { which } \\
\text { m } 100 \mathrm{n} \\
\text { pect to } \\
\text { reprodu }\end{array}$ & $\begin{array}{l}\text { referrec } \\
\text { termodu } \\
\text { se impe } \\
\text { given in } \\
\text { are lar } \\
\text { nm give } \\
\text { the valu } \\
\text { uce } C_{j}(\end{array}$ & $\begin{array}{l}\text { d to mix } \\
\text { lulation } \\
\text { edances } \\
\text { [28], [4 } \\
\text { rger than } \\
\text { en in [47 } \\
\text { ue provi } \\
\text { (0) giver }\end{array}$ & $\begin{array}{l}\text { xers as } \\
\text { produc } \\
\text { have } b \\
\text { 47] for } \\
\text { n the } \\
\text { 7] to } 8 \\
\text { ided in } \\
\text { en in lit }\end{array}$ & $\begin{array}{l}\text { s define } \\
\text { icts are } \\
\text { been co } \\
\mathrm{r} \text { the dic } \\
\text { values } \\
80 \mathrm{~nm} t \\
\mathrm{n} \text { [47] t } \\
\text { iterature } \\
\text { es fit of }\end{array}$ & $\begin{array}{l}\text { led by } \\
\text { provi } \\
\text { onsider } \\
\text { iodes ir } \\
\text { provid } \\
\text { to obta } \\
\text { to get } \\
\text { e for } \mathrm{t}\end{array}$ & $\begin{array}{l}\text { eqs. (3) } \\
\text { ided in } \\
\text { ered in } \\
\text { in the } 3 \\
\text { ded in t } \\
\text { tain } R_{s} \\
t \text { a bette } \\
\text { this dio } \\
\text { measure }\end{array}$ & $\begin{array}{l}\text { 3) and } \\
\text { [27], } \\
\text { our sir } \\
300-36 \\
\text { the lite } \\
\text { s,dc clc } \\
\text { er agre } \\
\text { ode. } \\
\text { ed dioc }\end{array}$ & $\begin{array}{l}\text { (4), respect } \\
\text { [46]: Z[3f } \\
\text { mulations. } \\
0 \mathrm{GHz} \mathrm{SH} \\
\text { rature for } \\
\text { oser to the } \\
\text { ement bety }\end{array}$ & $\begin{array}{l}f_{L O}-f_{R} \\
\text { tively, mixer (d } \\
\text { the LEC } \mathrm{n} \\
\text { values pro } \\
\text { ween meas } \\
\text { rve to the s }\end{array}$ & $\begin{array}{l}\text { cept for } \mathrm{t} \\
R F]=\mathrm{Z}[ \\
\text { doping, } \\
\text { model of } \\
\text { ovided ir } \\
\text { sured an }\end{array}$ & $\begin{array}{l}\text { the } 4 \\
{\left[f_{L O}\right.} \\
\text { lengt } \\
\text { f the } \\
\text { in the } \\
\text { ad sim }\end{array}$ & $\begin{array}{l}.75 \mathrm{~T} \\
+f \\
\text { th, an } \\
\text { diod } \\
\text { liter } \\
\text { mulate }\end{array}$ & $\begin{array}{l}\Gamma \mathrm{Hz} n \\
\mathrm{f}_{R F} \mathrm{C}= \\
\text { lode } \\
\text { le } \sim \\
\text { rature } \\
\text { ed co }\end{array}$ & $\begin{array}{l}\text { mixer that } \\
=Z\left[2 f_{L C}\right. \\
\text { area, barr } \\
11-15 \Omega \\
\text { onversion } \\
\text { liode equa }\end{array}$ & $\begin{array}{l}\text { nat are referre } \\
L O]=(6+28 \mathrm{j}) \\
\text { arrier height) } \\
\Omega \text {. The simul } \\
\text { n losses. } \\
\text { uation. } \\
\text { the } 300-360\end{array}$ & $\begin{array}{l}\text { ed to } \\
\Omega ; \\
\text { lead } \\
\text { lated }\end{array}$ \\
\hline
\end{tabular}

As an example, Fig. 4 shows the current noise spectra $S_{I}(f)$ for the 585-690 GHz fundamental mixer [34], see Table I, which lists the parameters of all the mixers analyzed in this work. Each noise spectrum in Fig. 4(a) can be divided into a low frequency region and a medium-high frequency region. The medium-high frequency region presents two peaks at frequencies around $3 \mathrm{THz}$ and $10 \mathrm{THz}$, see Fig. 4(a). These peaks are due to the evolution of the carrier density fluctuations originated in the transitions between the neutral and the depleted regions of the epilayer (first peak) and between the substrate and the epilayer (second peak), commonly known as returning carrier and hybrid plasma peaks, respectively [23], [24], [31], [41].

In Fig. 4(b), the low frequency region of the noise spectra in Fig. 4(a) are compared with the shot and thermal noise contributions given by the following equation [41], [43]:

$$
S_{I}(f \sim 0)=\frac{2 q I_{j} R_{j}^{2}}{\left(R_{s}+R_{j}\right)^{2}}+\frac{4 K_{B} T R_{s}}{\left(R_{s}+R_{j}\right)^{2}}
$$

where $R_{s}$ is the series resistance of the substrate and the non-depleted region of the epitaxial layer, $R_{j}$ represents the junction resistance and $I_{j}$ is the conduction current through the device. Although (6) is only valid under dc conditions [41], [43], it has been evaluated under the voltage waveforms obtained from MCHB using a lumped equivalent circuit (LEC) model that assumes $R_{s}(\mathrm{t}), R_{j}(\mathrm{t})$ and $I_{j}(\mathrm{t})$ in $(6)$. As a result, $S_{I}(f \sim 0)$ in (6) becomes time dependent, i.e., $S_{I}(f \sim 0, t)$. The red solid curve in Fig. 4(b) is the time average of $S_{I}(f \sim 0, t)$ in an IF period for each LO power. A good agreement is observed between $\mathrm{MC}$ and the time average of this analytical model.

The LEC models used in this work are based on the LEC of the Schottky junction in series with a series resistance. The non-

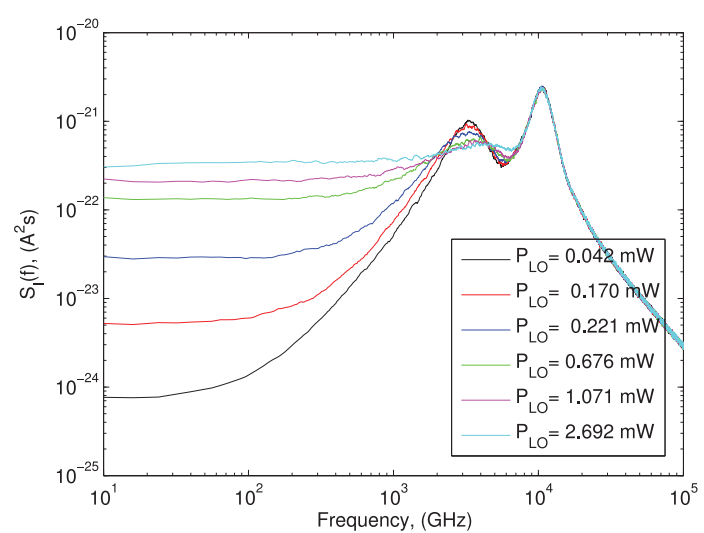

(a)

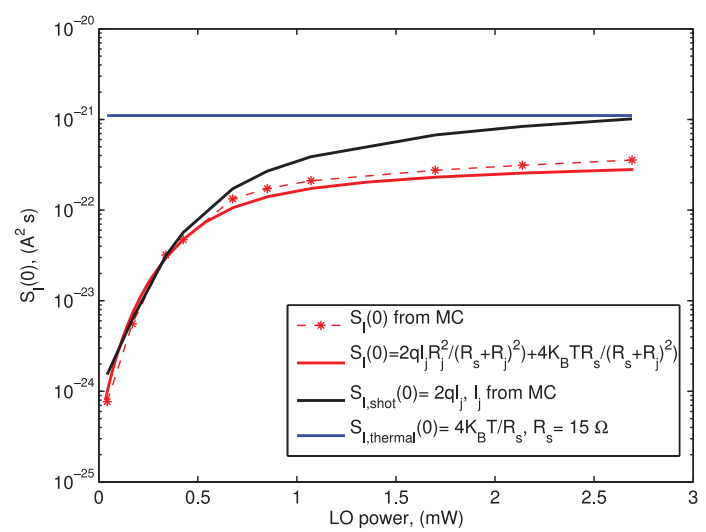

(b)

Fig. 4. (a) Noise spectra of the current fluctuations at the terminals of the diode in the 585-690 GHz fundamental mixer [34], [42], see Table I, under the voltage waveforms obtained with $\mathrm{MCHB}$ for $f_{\mathrm{RF}}=585 \mathrm{GHz}$ and $f_{\mathrm{LO}}=580$ GHz. (b) Comparison of the low frequency current noise spectra obtained with MC model in (a) and the shot and thermal noise contribution evaluated with $\mathrm{LEC}_{R_{s}(t)}$. The junction resistance is time varying in the LEC model $R_{j}(\mathrm{t})$. 
TABLE II

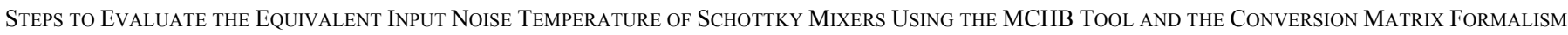

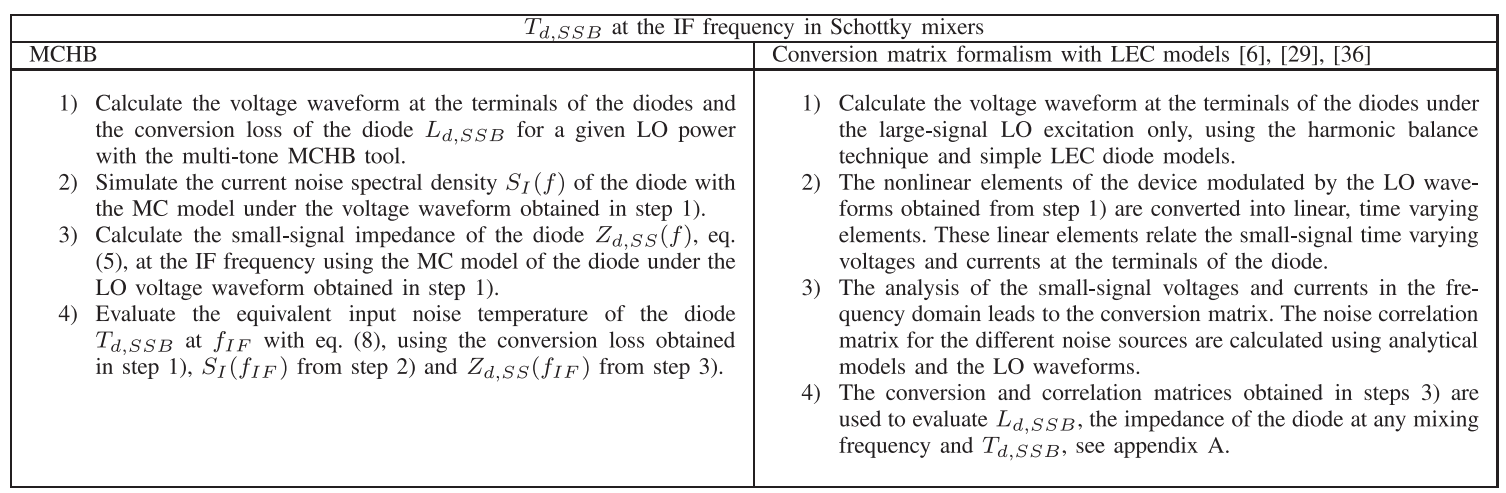

linear capacitance is modeled with a fully depleted approximation and the conduction current through the Schottky contact is approached by thermionic emission equation [44]. Under time varying conditions, both the nonlinear capacitance, $C_{j}(t)$, and the nonlinear resistance, $R_{j}(t)$, are assumed time varying in the LEC models. Diode models with the dc series resistance of the diode $R_{s, \mathrm{dc}}$ (the series resistance measured when the epilayer is undepleted) and with the time varying series resistance $R_{s}(t)$ due to the time variation of the width of the depletion region [5], [45] have been used. These model are denoted by $\mathrm{LEC}_{R_{s, \mathrm{dc}}}$ and $\mathrm{LEC}_{R_{s}(t)}$ respectively. Also, a LEC model which takes into account carrier inertia and displacement current in undepleted regions of the device by means of an inductance and a capacitance [7], respectively, has been considered $\left(\mathrm{LEC}_{R_{s} \mathrm{LC}(t)}\right.$ model).

2) Calculation of $Z_{d, \mathrm{SS}}$ : The impedance $Z_{d, \mathrm{SS}}$ depends on the LO signal and the bias point. It also depends on the embedding impedances of the mixer circuit at frequencies different from the frequency at which $Z_{d, \mathrm{SS}}$ is evaluated. $Z_{d, \mathrm{SS}}$ can be calculated directly according to its definition in (5): The diode is excited with the $\mathrm{LO}$ voltage waveforms at the terminals of the diode obtained from the MCHB simulations and a small-signal sinusoidal voltage at the IF frequency- $V_{\mathrm{SS}}\left(f_{\mathrm{IF}}\right)$-superimposed to the LO voltage waveform. The contribution to the current response of the diode is evaluated and the small-signal impedance at the IF frequency $Z_{d, \mathrm{SS}}\left(f_{\mathrm{IF}}\right)$ is calculated using (5).

Fig. 5 presents a comparison of $Z_{d, \mathrm{SS}}\left(f_{\mathrm{IF}}\right)$ obtained with MCHB and the output impedance of the pumped diode $Z_{\text {out }}\left(f_{\mathrm{IF}}\right)$ defined in the conversion matrix (CM) formalism [6], [29], see Appendix A, for the diode in the $585-690 \mathrm{GHz}$ fundamental mixer. A good agreement is observed between $Z_{d, \mathrm{SS}}\left(f_{\mathrm{IF}}\right)$ and $Z_{\text {out }}\left(f_{\mathrm{IF}}\right)$ from the $\mathrm{CM}$ formalism using $\mathrm{LEC}_{R_{s, \mathrm{dc}}}$ and $\mathrm{LEC}_{R_{s}(t)}$ models. The real part of these impedances decreases as the LO power increases because the nonlinear resistance of the Schottky junction decreases as the diode approaches flat band conditions. On the other hand, the performance of the imaginary part is determined by the nonlinear capacitance of the diode (increasing the input power, the swept of the nonlinear capacitance increases until it reaches flat-band conditions).

3) Evaluation of the Equivalent Input Noise Temperature: Once $S_{I}\left(f_{\mathrm{IF}}\right)$ and $Z_{d, \mathrm{SS}}\left(f_{\mathrm{IF}}\right)$ have been calculated, the noise power delivered to the output impedance of the mixer circuit at

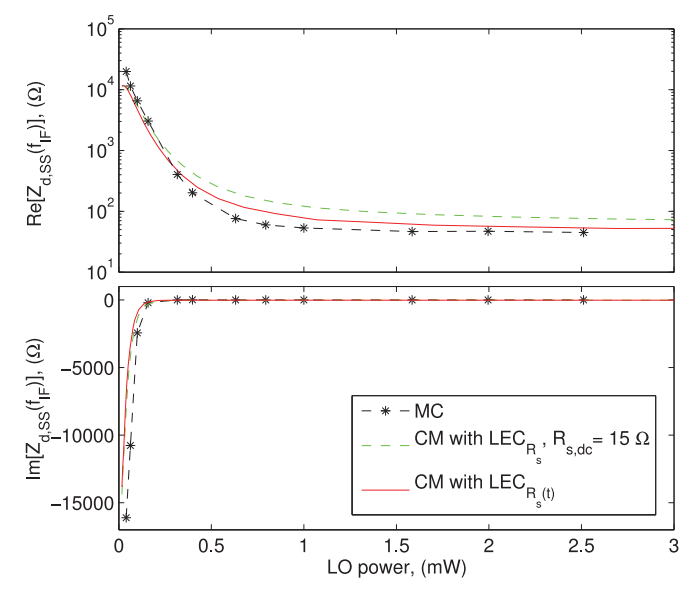

Fig. 5. Real and imaginary parts of the impedances $Z_{d, \mathrm{SS}}\left(f_{\mathrm{IF}}\right)$ calculated with $\mathrm{MCHB}$ and $Z_{\mathrm{out}}\left(f_{\mathrm{IF}}\right)$ defined in the conversion matrix formalism, see Appendix A, for the diode in the $585-690 \mathrm{GHz}$ fundamental mixer at IF frequency $5 \mathrm{GHz}$.

$f_{\mathrm{IF}}, Z_{e}\left(f_{\mathrm{IF}}\right)$, see Fig. 3 , is given by the following expression, obtained from the analysis of the circuit in Fig. 3(b):

$$
P_{\mathrm{del}}\left(f_{\mathrm{IF}}\right)=\frac{S_{I}\left(f_{\mathrm{IF}}\right) \Delta f \cdot \operatorname{Re}\left[Z_{e}\left(f_{\mathrm{IF}}\right)\right] \cdot\left|Z_{d, \mathrm{SS}}\left(f_{\mathrm{IF}}\right)\right|^{2}}{\left|Z_{d, \mathrm{SS}}\left(f_{\mathrm{IF}}\right)+Z_{e}\left(f_{\mathrm{IF}}\right)\right|^{2}}
$$

where $\Delta f$ is the bandwidth of interest. The single sideband equivalent input noise temperature of the diode is defined as [6], [29]

$$
T_{d, \mathrm{SSB}}=\frac{P_{\mathrm{del}} L_{d, \mathrm{SSB}}}{k_{B} \Delta f}
$$

where $L_{d, \mathrm{SSB}}$ is the single sideband conversion loss of the diode between the RF and the IF signals. Once the single sideband noise temperature of the diode is calculated, the double sideband noise temperature of the mixer and the receiver can be evaluated using (4) and (2), respectively. Table II shows a summary of the procedure to calculate the equivalent input noise temperature of Schottky mixers with the MCHB tool and the CM formalism.

\section{Validation of the MiXer Simulation ToOl}

The validation of the MCHB tool has been carried out by comparing simulation results with measurements published in 


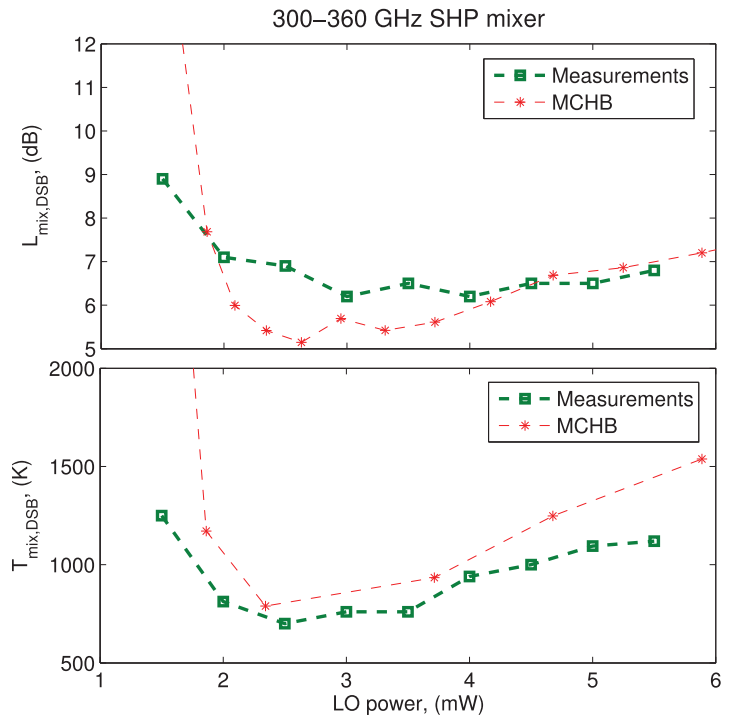

Fig. 6. DSB conversion losses equation (3) and noise temperature equation (4) of the $300-360 \mathrm{GHz}$ SHP mixer [47] as a function of the LO power.

the literature for Schottky mixers, see Table I. Mixers are usually measured at the available LO power, and hence, most of the publications only present measurements for a fixed LO power. The circuit impedances at the LO, RF and IF frequencies in the literature have been used in the simulations. The IF frequencies considered in our simulations have been chosen higher than those used in measurements to decrease the computational cost of the MCHB, see Table I. However, it has been tested that these differences have a low impact on the simulation results (see the results in Section IV-A for the $2500 \mathrm{GHz}$ mixer at IF frequencies 5 and $25 \mathrm{GHz}$ ).

Fig. 6 shows a good agreement between measured and simulated results of $L_{\mathrm{mix}, \mathrm{DSB}}$ and $T_{\mathrm{mix}, \mathrm{DSB}}$ for the $300-360 \mathrm{GHz}$ SHP mixer [47], see Table I.

A comparison between measured and simulated results for $L_{\text {rec,DSB }}$ and $T_{\text {rec,DSB }}$ of the $585-690 \mathrm{GHz}$ fundamental mixer in [34] is shown in Fig. 7, see Table I. Since the bias voltage $V_{\text {bias }}$ of this mixer is not provided in the literature ${ }^{3}, V_{\text {bias }}=0.6$ $\mathrm{V}$ and $0.75 \mathrm{~V}$ have been simulated with the MCHB tool. By increasing the bias point, the LO power to achieve the minimum conversion losses is lower. Besides, the minimum conversion losses are higher for higher bias due to the lower modulation of the nonlinear resistance of the Schottky diode. The noise temperature is lower when $V_{\text {bias }}$ is $0.6 \mathrm{~V}$ since the conversion losses with this $V_{\text {bias }}$ are lower.

A good agreement is observed between measurements and simulations in Fig. 7. Additional measured results for a $585 \mathrm{GHz}$ fundamental mixer similar to that in [34] have been presented in $[54]^{4}$, with a minimum receiver noise temperature of $1700 \mathrm{~K}$. This noise temperature is in good agreement with the minimum $T_{\text {rec,DSB }}$ obtained with MCHB for $V_{\text {bias }}=0.6 \mathrm{~V}$, see Fig. 7.

The 1.2 THz SHP mixer described in [51] has been simulated with our MCHB tool, see Fig. 8. Measured data for the designs $A$ and $B$ presented in [51] are included in this figure. According

\footnotetext{
${ }^{3}$ According to [34], bias voltages used in measurements were optimized at each LO power to minimize the noise temperature.

${ }^{4}$ The epilayer doping concentration of the diode in [54] is $4 \times 10^{17} \mathrm{~cm}^{-3}$, while for the diode in [34] is $2 \times 10^{17} \mathrm{~cm}^{-3}$.
}

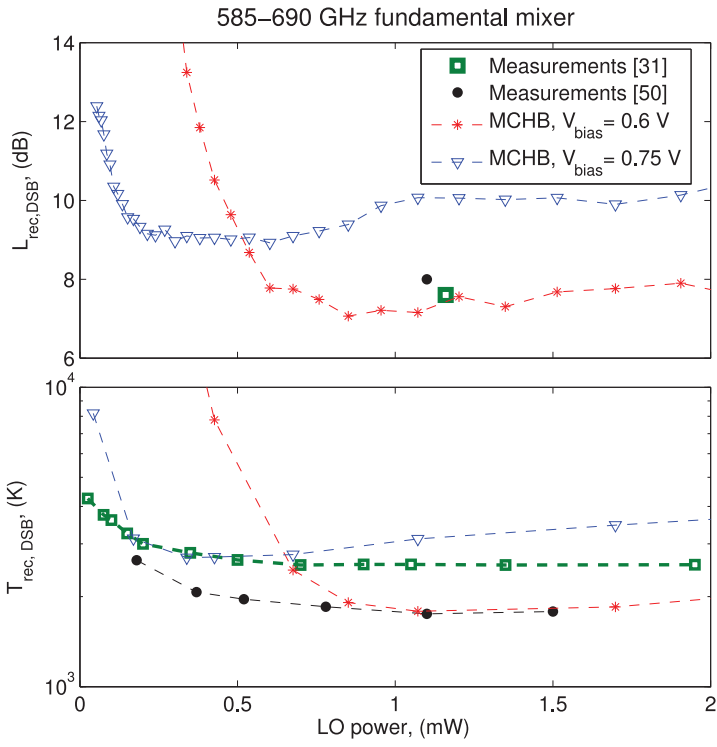

Fig. 7. DSB conversion losses equation (1) and noise temperature equation (2) of the 585-690 GHz fundamental receiver [34] as a function of the LO power.

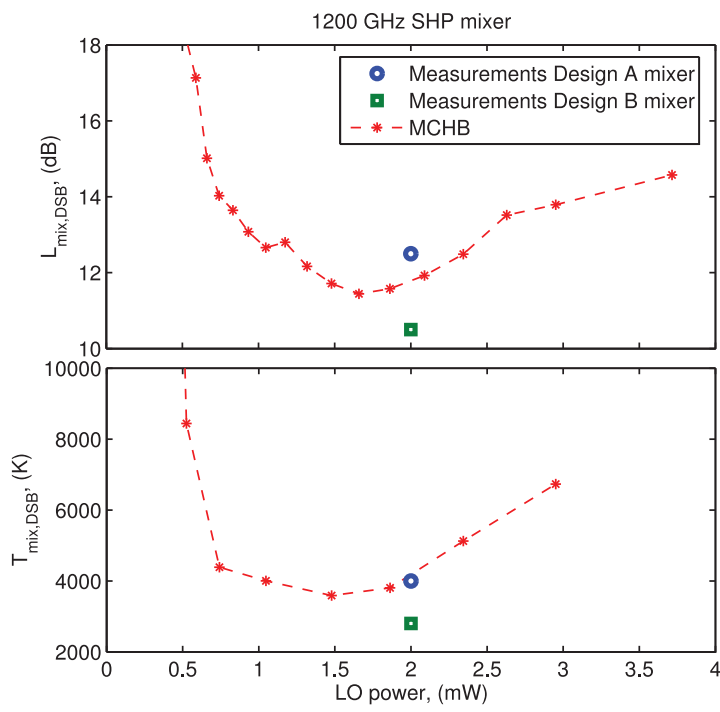

Fig. 8. DSB conversion losses equation (3) and noise temperature equation (4) of the $1200 \mathrm{GHz}$ SHP mixer [51] as a function of the LO power.

to [51], mixer design A was optimized to provide an operating bandwidth from 1080 to $1280 \mathrm{GHz}(17 \%)$, and mixer design $\mathrm{B}$ was optimized to provide the highest possible sensitivity in the 1120-1280 GHz band. The parameters used for the simulation of this mixer are shown in Table I. Due to the lack of information in [51], the following strategy has been used in the simulation of this mixer.

- The parameters of the diodes in [55] for a $874 \mathrm{GHz} \mathrm{SHP}$ mixer have been adopted for the diodes in the $1.2 \mathrm{THz} \mathrm{SHP}$ mixer. This was indicated in [50] for the design of a 1.2 $\mathrm{THz}$ mixer.

- The circuit impedances at the RF, LO and IF frequencies have been optimized with the MCHB tool at LO power 2 $\mathrm{mW}$. A parasitic capacitance of $5 \mathrm{fF}$ has been considered.

- Losses $L_{\mathrm{qo}}=0 \mathrm{~dB}, L_{\mathrm{rf}}=0.7 \mathrm{~dB}$, and $L_{\mathrm{if}}=1.2 \mathrm{~dB}$ given in [48], [49] for the 835-900 GHz fundamental balanced mixer have been assumed. 


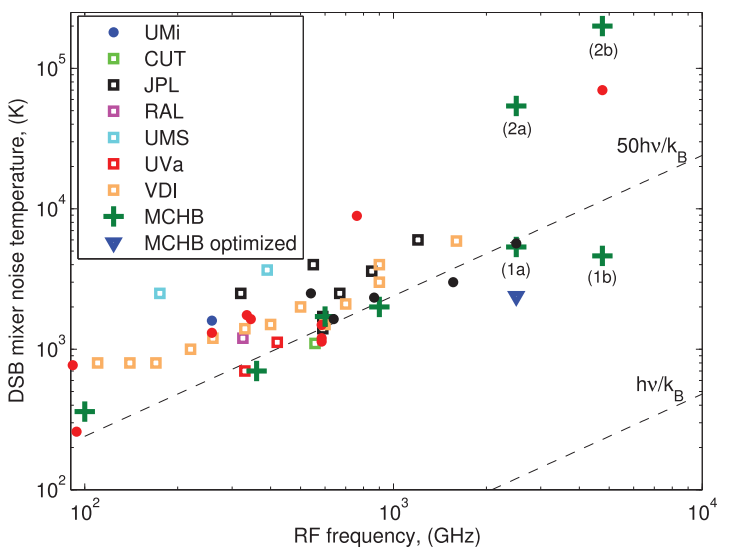

(a)

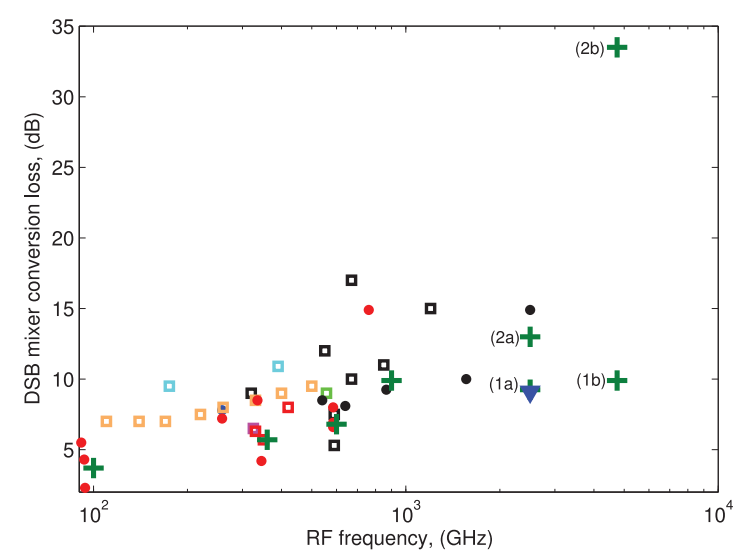

(b)

Fig. 9. Main results of measured (a) $T_{\text {mix,DSB }}$ and (b) $L_{\text {mix }, \text { DSB }}$ for fundamental (solid circles $\bullet$ ) and SHP (squares $\square$ ) planar Schottky mixers available in the literature since 1991. Measurements and MCHB simulation results for the mixers in Table I are included in this figure. The $4.75 \mathrm{THz}$ mixer is based on a whisker diode and the measurements are for the receiver instead. The data in the legend indicate the laboratory which built the diodes: CUT: Chalmers University of Technology. JPL: Jet Propulsion Laboratory. RAL: Rutherford Appelton Laboratory. UMi: University of Michigan. UMS: United Monolithic Semiconductor. UVa: University of Virginia. VDI: Virginia Diodes, Inc.

Simulation results in Fig. 8 show a good agreement with the measurements provided in [51].

An additional validation of the MCHB tool is shown in Fig. 9, which presents the main results in the literature for $L_{\text {mix, DSB }}$ and $T_{\text {mix,DSB }}$ of planar Schottky mixers together with MCHB simulation results for the mixers in Table I. At RF frequencies below $2.5 \mathrm{THz}$, MCHB simulation results show a good agreement with measurements. For the $2.5 \mathrm{THz}$ mixer, the minimum values of $L_{\mathrm{mix}, \mathrm{DSB}}$ and $T_{\mathrm{mix}, \mathrm{DSB}}\left[\mathrm{P}_{\mathrm{LO}} \sim 5 \mathrm{~mW}\right.$, denoted by (1a)] as well as those obtained at the available LO power $\left[\mathrm{P}_{\mathrm{LO}}=6 \mathrm{~mW}\right.$, denoted by (2a) $]$ are indicated, see Fig. 10. Also, simulated results from the optimization of this mixer are included. Simulation results for the $4.75 \mathrm{THz}$ fundamental mixer in [53] are also included in Fig. 9. Since $L_{\mathrm{op}}, L_{\mathrm{rf}}$ and $L_{\mathrm{if}}$ are not published for this mixer, those given for the $2.5 \mathrm{THz}$ mixer have been considered in the simulations. As for the $2.5 \mathrm{THz}$ mixer, minimum values of simulated $L_{\mathrm{rec}, \mathrm{DSB}}$ and $T_{\text {rec,DSB }}$ [denoted by (1b)] and the values obtained at the available input power $\left[\mathrm{P}_{\mathrm{LO}}=1 \mathrm{~mW}\right.$, denoted by (2b)] are shown, see Fig. 14 . The performance of these mixers and mixers above $2.5 \mathrm{THz}$ are analyzed in Section IV.

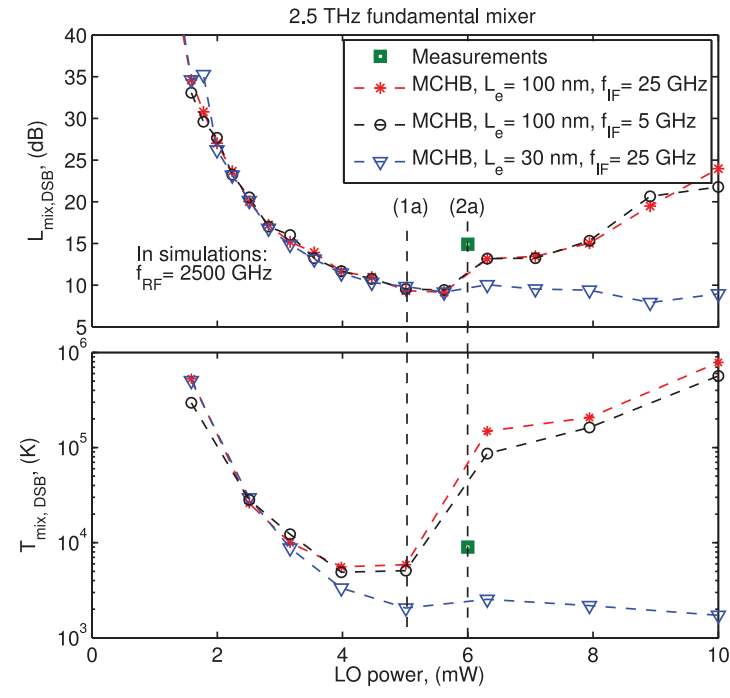

Fig. 10. DSB conversion loss and noise temperature of the $2.5 \mathrm{THz}$ fundamental mixer-(3) and (4) - described in Table I with $L_{e}=100$ and $30 \mathrm{~nm}$, obtained with MCHB. IF frequencies 5 and $25 \mathrm{GHz}$ have been simulated to justify the use of high IF frequencies in simulations, as discussed at the beginning of Section III.

\section{ANAlysis OF SCHOtTKy MiXers ABOVE $2 \mathrm{THz}$}

The analysis and design of Schottky mixers require reliable simulation tools to account for the physical effects which determine the performance of the devices. For high RF frequencies, the electrical and noise responses of the Schottky diodes are limited by physical effects like velocity saturation, plasma resonance and nonlocal effects both in time and space. An analysis of the impact of these phenomena in the performance of Schottky mixers is carried out in this section using the MCHB tool.

\section{A. Results for a $2.5 \mathrm{THz}$ Fundamental Mixer}

Fig. 10 shows the DSB conversion losses and the equivalent input noise temperature of the $2.5 \mathrm{THz}$ fundamental mixer in [52] as a function of the LO power, see Table I. Two values of the epilayer length $L_{e}$ have been simulated: $100 \mathrm{~nm}$ according to [52], and $30 \mathrm{~nm}$, in order to optimize the performance of the mixer. Lines (1a) and (2a) indicate the values included in Fig. 9.

MCHB simulation results show an abrupt increase of the conversion losses at $\mathrm{P}_{\mathrm{LO}} \sim 6 \mathrm{~mW}$ (flat band conditions are achieved at $\mathrm{P}_{\mathrm{LO}} \sim 4 \mathrm{~mW}$ ) when $L_{e}=100 \mathrm{~nm}$. This performance is due to velocity saturation effects originated by the increase of the occupation of the lower mobility upper valleys $(L, X)$ in the undepleted region of the epilayer of the diode. MCHB shows occupations of the $\mathrm{L}$ valley around $5 \%$ at $P_{\mathrm{LO}}=2.5 \mathrm{~mW}$, reaching $\sim 20 \%$ at $5 \mathrm{~mW}$; at higher $\mathrm{LO}$ power, the population of the $\mathrm{L}$ valley increases quickly, with maximum occupation around $60 \%$ at $P_{\mathrm{LO}} \sim 6.3 \mathrm{~mW}$. Also, the transfer to the $\mathrm{X}$ valley increases abruptly when the $\mathrm{LO}$ power increases from 5 to $6.3 \mathrm{~mW}$, rising from occupations lower than $5 \%-20 \%$, see Fig. 11.

The noise temperature of the mixer is also degraded when the LO power is higher than $4 \mathrm{~mW}$, see Fig. 10. As the electric field in the undepleted epilayer increases when the LO power increases, the energy of the electrons is increased above the equilibrium thermal energy. This effect increases the diode noise 


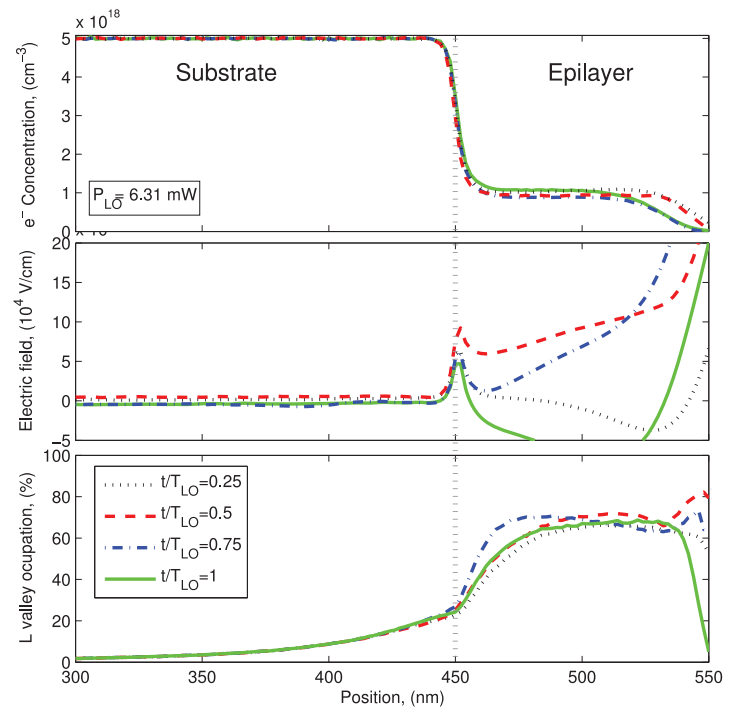

Fig. 11. Profiles of the electron concentration, the electric field, and the occupation of the $\mathrm{L}$ valley (percentage of electrons in the $\mathrm{L}$ valley) for the $2.5 \mathrm{THz}$ mixer with $\mathrm{L}_{e}=100 \mathrm{~nm}$, obtained with $\mathrm{MC}$ at different times in a LO period $\left(T_{\mathrm{LO}}\right)$.

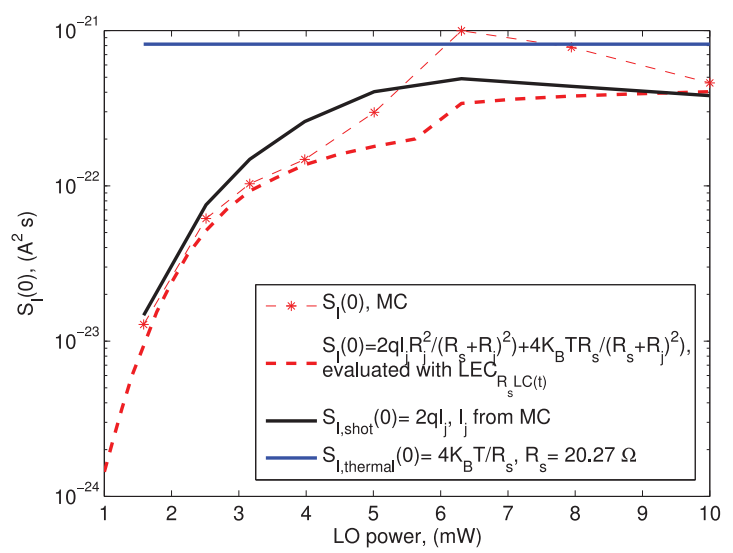

Fig. 12. Comparison of the low frequency region of the current noise spectra obtained with the $\mathrm{MC}$ model for the diode in the $2.5 \mathrm{THz}$ mixer under the voltage waveforms from MCHB for different $\mathrm{LO}$ powers. The shot and thermal noise contribution of (6) evaluated with a $\mathrm{LEC}_{R_{s} L C(t)}$ model using the voltage waveforms obtained with MCHB is also included. The dc component of the current $I_{0}$ in the $2 q I_{0}$ law has been obtained with the MC model.

temperature, giving rise to hot electron noise [14], [15]. When the electric field is high enough for intervalley transfer to take place, the stochastic transitions between the lowest and upper valleys of the semiconductor generate a new contribution to the noise spectrum usually known as intervalley scattering noise [14], [22]. For the mixer with $L_{e}=100 \mathrm{~nm}$ in Fig. 10, an abrupt increase of the noise temperature is observed at $P_{\mathrm{LO}}$ around 6 $\mathrm{mW}$ due to the quick increase of the intervalley transfer at these LO powers. The increase of noise due to hot electrons and intervalley transfers at $P_{\mathrm{LO}}$ higher than $4 \mathrm{~mW}$ is observed in Fig. 12, where the low frequency region of the current noise spectra obtained with the MC model for this mixer is compared with (6) assumed time varying for the combined contribution of shot and thermal noise. In this figure, (6) is evaluated with a $\mathrm{LEC}_{R_{s} L C(t)}$ model under the voltage waveforms obtained from the MCHB simulations.

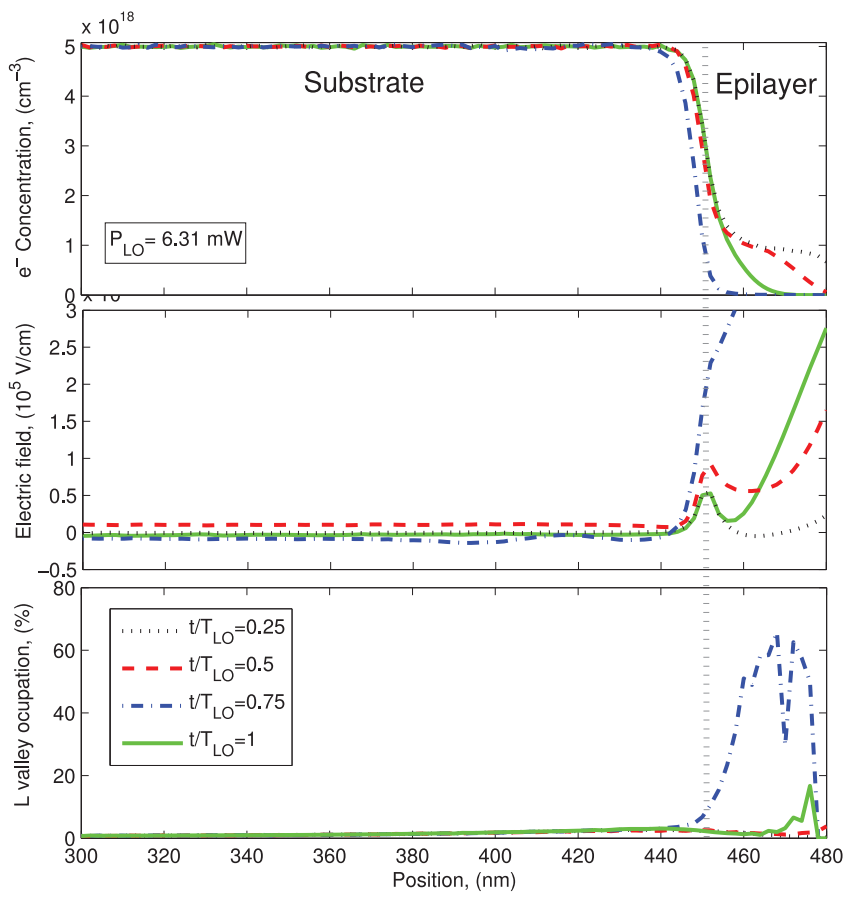

Fig. 13. Profiles of the electron concentration, the electric field, and the occupation of the $\mathrm{L}$ valley for the $2.5 \mathrm{THz}$ mixer with $\mathrm{L}_{e}=30 \mathrm{~nm}$, obtained with $\mathrm{MC}$ at different times in a LO period $\left(T_{\mathrm{LO}}\right)$.

In order to optimize the performance of this mixer, the epilayer length $L_{e}$ is set to $30 \mathrm{~nm}$ (the epilayer length of the diode in the $4.75 \mathrm{THz}$ mixer, see Table I). The abrupt increase of the conversion losses and the noise temperature observed in Fig. 10 for $L_{e}=100 \mathrm{~nm}$ disappears. In the optimized diode, the epilayer is totally depleted during a high temporal fraction of a LO period, see Fig. 13, what mitigates velocity saturation effects. Besides, nonlocal transport effects [11], [30] are important in the performance of this diode. The electrons in the substrate (low field region) enter into the epilayer (high field region), where they move with a velocity higher than expected from the equilibrium velocity field curve. In addition, the mean free path of the electrons in the epilayer is of the order of the epilayer length $\left(L_{e}=30 \mathrm{~nm}\right)$, and ballistic transport is important. Due to these effects, most of the electrons remain in the lower valley (according to MCHB, the occupation of the upper valleys remains lower than $5 \%$ when $L_{e}=30 \mathrm{~nm}$ for the simulated power swept), see Fig. 13. Therefore, the conversion losses and the noise temperature remain nearly constant at $P_{\mathrm{LO}}$ higher than 4 $\mathrm{mW}$ for $L_{e}=30 \mathrm{~nm}$, in contrast to the abrupt increase of these parameters observed for the diode with $L_{e}=100 \mathrm{~nm}$ at $P_{\mathrm{LO}}$ around $6 \mathrm{~mW}$, see Fig. 10.

\section{B. Results for a $4.75 \mathrm{THz}$ Fundamental Mixer}

The 4.75 THz mixer in [53] is based on a Schottky diode with $L_{e}=30 \mathrm{~nm}$, see Table I. Fig. 14 shows the conversion losses and the noise temperature obtained from the MCHB simulations of this mixer (since $L_{\mathrm{op}}, L_{\mathrm{rf}}$ and $L_{\mathrm{if}}$ are not provided for the $4.75 \mathrm{THz}$ mixer, those given for the $2.5 \mathrm{THz}$ mixer in [52] have been used to evaluate $L_{\text {rec }}$ and $T_{\text {rec }}$ ). Lines (1b) and (2b) indicate the values included in Fig. 9. Intervalley transfer effects are highly mitigated due the short length of the epilayer of the diode, as was described for the for the $2.5 \mathrm{THz}$ mixer 


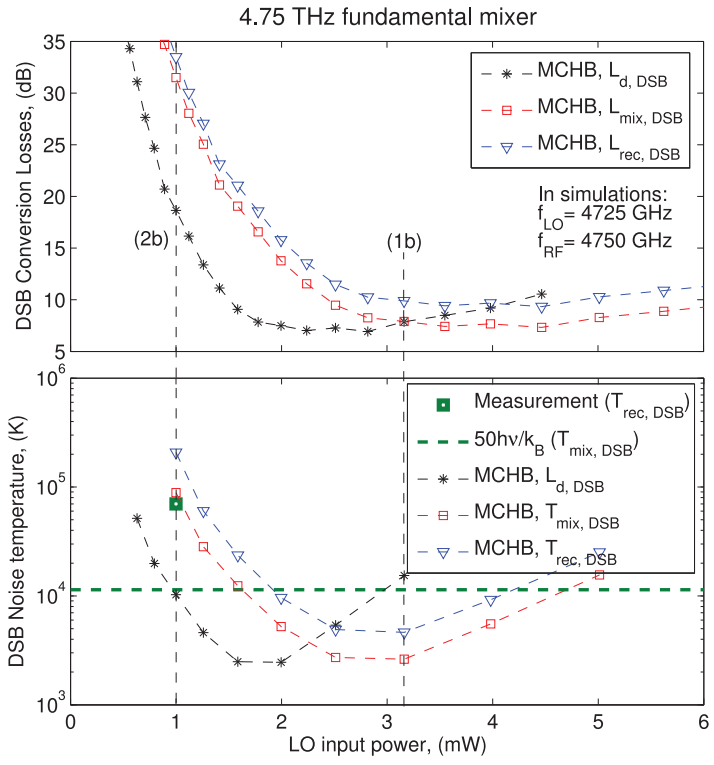

Fig. 14. DSB Conversion losses and noise temperature of the $4.75 \mathrm{THz}$ fundamental mixer described in Table I, obtained with MCHB at $f_{\mathrm{IF}}=25 \mathrm{GHz}$. Results for the diode (black lines), the mixer (red lines) and the receiver (blue lines) are presented. $L_{\mathrm{op}}, L_{\mathrm{rf}}$ and $L_{\mathrm{if}}$ of the $2.5 \mathrm{THz}$ mixer have been used to evaluate the mixer and the receiver characteristics.

with $L_{e}=30 \mathrm{~nm}$. The simulated $T_{\mathrm{rec}, \mathrm{DSB}}$ at the available LO power $\sim 1 \mathrm{~mW}$ is of the order of the measured results. Simulations indicate that higher LO power is required to get lower noise temperature. Accurate information about the real losses $L_{\mathrm{op}}, L_{\mathrm{rf}}$ and $L_{\mathrm{if}}$ of this mixer will provide a better interpretation of the measured receiver noise temperature. On the other hand, the $4.75 \mathrm{THz}$ mixer in [53] is an old design based on whisker technology.

\section{Schottky Mixers up to $10 \mathrm{THz}$}

According to the results in previous subsections, a short epilayer length is necessary to mitigate intervalley transfer effects. Hence, in this section, an analysis of Schottky mixers up to signal frequency $10 \mathrm{THz}$ is carried out, using as a reference the diode of the $4.75 \mathrm{THz}$ fundamental mixer [53], Table I, characterized by an epilayer of length $30 \mathrm{~nm}$, doping concentration $1 \times 10^{18} \mathrm{~cm}^{-3}$, anode area $0.07 \mu \mathrm{m}^{2}\left(C_{j}(0)=0.25 \mathrm{fF}\right)$ and series resistance $40 \Omega$. In the simulation of these mixers, the circuit impedances at the RF and LO frequencies have been conjugately matched for each $L O$ power considered. The IF impedances $Z_{\mathrm{IF}}$ have been set to $250 \Omega$ for all the designs. $L_{\mathrm{qo}}=1$ $\mathrm{dB}, L_{\mathrm{rf}}=2 \mathrm{~dB}$, and $L_{\mathrm{if}}=1 \mathrm{~dB}$ have been assumed. These are reasonable losses for the $\mathrm{THz}$ frequencies considered in this subsection.

Fig. 15 shows the mixer conversion losses of the $2.5 \mathrm{THz}$, $4.75 \mathrm{THz}$ and $10 \mathrm{THz}$ fundamental mixers based on the reference diode in this subsection, obtained with MCHB. When the signal frequency is increased, both the minimum conversion losses and the LO power to achieve these minimum conversion losses increase. This degradation of the mixer performance is due to the shunting effect of the junction capacitance $\left(C_{j}\right)$ over the junction resistance $\left(R_{j}\right)$ of the diode [56]. At high frequencies, smaller anode areas are beneficial since they contribute to

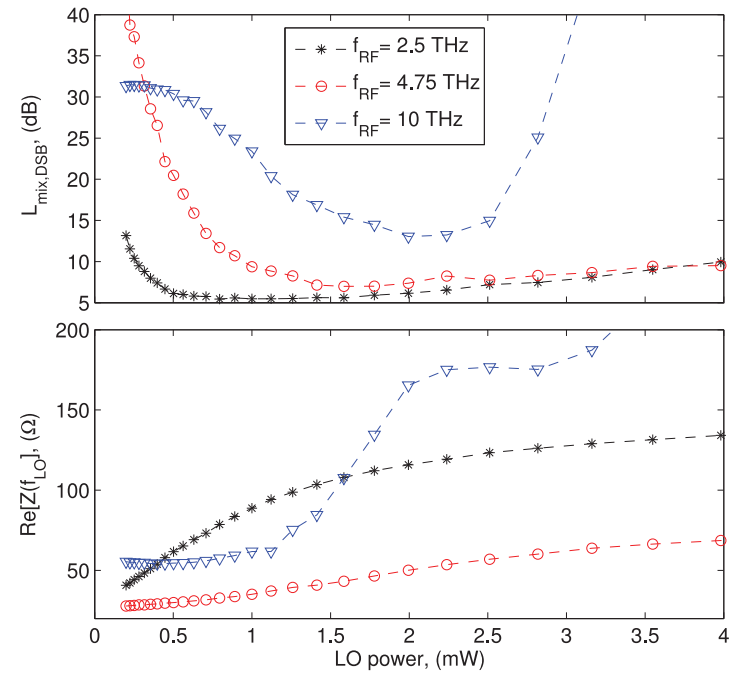

Fig. 15. Comparison of the mixer conversion losses and the real part of the diode impedance at the LO frequency as a function of the LO power for the $2.5,4.75$, and the $10 \mathrm{THz}$ fundamental mixers based on the reference diode $\left(f_{\mathrm{IF}}=25 \mathrm{GHz}\right)$. The anode area of the diode is $0.07 \mu \mathrm{m}^{2}$. Results obtained with MCHB.

achieve the minimum conversion losses at lower LO powers. On the other hand, smaller anode areas at these frequencies are beneficial to obtain easy to match mixers [56].

Fig. 15 shows the real part of the diode impedance $Z_{d}$ at the LO frequency as a function of the LO power for the mixers analyzed in this subsection. When the signal frequency is increased from 2.5 to $4.75 \mathrm{THz}$, the real part of the diode impedance decreases due to the shunting of the nonlinear capacitance. When the signal frequency is increased to $10 \mathrm{THz}, \operatorname{Re}\left[Z_{d}\right]$ at low LO power increases due to plasma effects in the undepleted epilayer (plasma frequency of the epilayer is around $9.6 \mathrm{THz}$, see [7]). At LO power higher than $1 \mathrm{~mW}$, the occupation of the upper valleys of the $10 \mathrm{THz}$ mixer increases quickly, resulting in the abrupt increase of $\operatorname{Re}\left[Z_{d}\right]$ observed in Fig. 15. The MC simulations of this mixer show that, at $P_{\mathrm{LO}}=1 \mathrm{~mW}$, the maximum occupations of the $\mathrm{L}$ and $\mathrm{X}$ valleys in the undepleted regions of the diode are around $27 \%$ and $1.5 \%$, respectively, and at $P_{\mathrm{LO}}=1.4 \mathrm{~mW}$ they are around $46 \%$ and $5 \%$ (for comparison, at $P_{\mathrm{LO}}=3 \mathrm{~mW}$, the occupations of the $\mathrm{L}$ and X valleys in the $2.5 \mathrm{THz}$ mixer are lower than $12 \%$ and $3 \%$, respectively, and for the $4.75 \mathrm{THz}$ mixer they are lower than $25 \%$ and $5 \%$.).

The equivalent input noise temperature of the mixer $T_{\text {mix,DSB }}$ and the equivalent noise temperature of the diode $T_{\text {noise }, ~}{ }^{5}$ in the $2.5 \mathrm{THz}, 4.75 \mathrm{THz}$ and the $10 \mathrm{THz}$ fundamental mixers based on the reference diode of this subsection are shown in Fig. 16. For the 2.5 and $4.75 \mathrm{THz}$ mixers and up to LO powers around $1.3 \mathrm{~mW}, T_{\text {noise },} d$ increases due to shot and thermal noise contributions. At higher LO powers, hot electron noise and intervalley transfer noise lead to a further increase of $T_{\text {noise, } d}$. These noise components are more important in the $4.75 \mathrm{THz}$ mixer because the electron energy and the occupation of the $\mathrm{L}$ valley are higher. For the $10 \mathrm{THz}$ mixer, hot electron noise and intervalley transfer noise are important at $P_{\mathrm{LO}}=1 \mathrm{~mW}$, leading to

${ }^{5} T_{\text {noise, } d}$ is not referred to the input, i.e., it represents the noise power of the diode delivered to the IF load, $T_{\text {noise }, d}=P_{\text {del }} / k_{B} \Delta f$, see (8). 


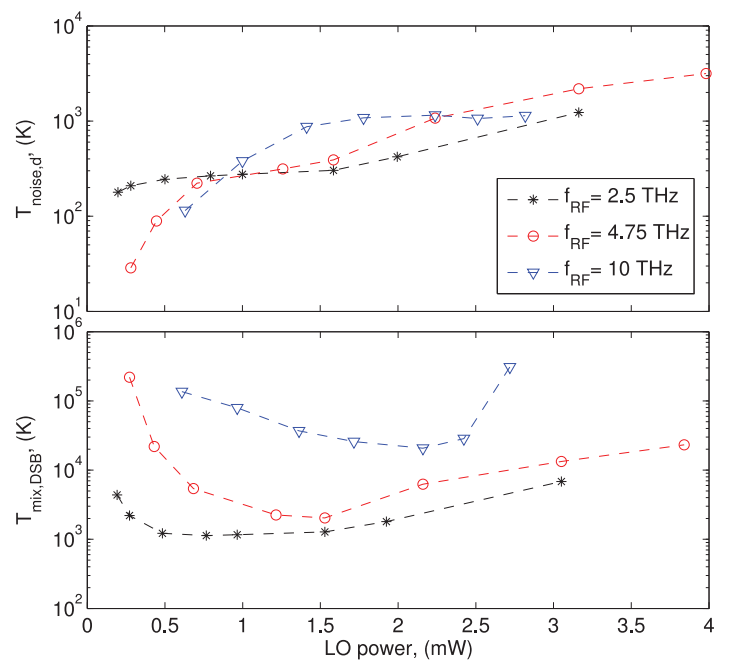

Fig. 16. Equivalent noise temperature $T_{\text {noise }, d}$ of the diode and double side band equivalent input noise temperature $T_{\mathrm{mix}, \mathrm{DSB}}$ of the reference $2.5 \mathrm{THz}$, $4.75 \mathrm{THz}$ and the $10 \mathrm{THz}$ fundamental mixers, obtained with MCHB.

TABLE III

Optimum Values of $N_{e}$ AND $L_{e}$ OBtained With THE MCHB ToOl FoR MiXers Above 1 THz. THE Minimum $L_{\text {mix }}$ AND $T_{\text {mix }}$ AS WeLl AS the Values at the Available lo Power Are Shown. The Results Using THE DESIGNS IN TABLE I ARE INCLUDED FOR COMPARISON

\begin{tabular}{|c|c|c|c|c|}
\hline \multicolumn{5}{|c|}{ Optimum designs } \\
\hline Mixer & $\begin{array}{c}N_{e} \\
\left(\mathrm{~cm}^{-3}\right)\end{array}$ & $\begin{array}{c}L_{e} \\
(\mathrm{~nm})\end{array}$ & $\begin{array}{c}L_{m i x}{ }^{\mathrm{a}} \\
(\mathrm{dB}, \mathrm{DSB})\end{array}$ & $\begin{array}{c}T_{m i x}{ }^{\mathrm{a}} \\
(\mathrm{K}, \mathrm{DSB})\end{array}$ \\
\hline $1200 \mathrm{GHz}$ subharmonic & $5 \times 10^{17}$ & 50 & $8.7-10.35$ & $1510-2890$ \\
\hline 2500 GHz fundamental & $1 \times 10^{18}$ & 30 & $4.1-5.6$ & $610-810$ \\
\hline 4750 fundamental & $2 \times 10^{18}$ & 30 & $4.2-7.8$ & $1190-1720$ \\
\hline \multicolumn{5}{|c|}{ Designs from Table I } \\
\hline Mixer & $\begin{array}{c}N_{e} \\
\left(\mathrm{~cm}^{-3}\right)\end{array}$ & $\begin{array}{c}L_{e} \\
(\mathrm{~nm})\end{array}$ & $\begin{array}{c}L_{m i x}{ }^{\mathrm{a}} \\
(\mathrm{dB}, \mathrm{DSB})\end{array}$ & $\begin{array}{c}T_{m i x}{ }^{\mathrm{a}} \\
(\mathrm{K}, \mathrm{DSB})\end{array}$ \\
\hline 1200 GHz subharmonic & $5 \times 10^{17}$ & 100 & 11.5 & 3590 \\
\hline 2500 GHz fundamental & $1 \times 10^{18}$ & 100 & $9.25-13$ & $5350-54000$ \\
\hline 4750 fundamental & $1 \times 10^{18}$ & 30 & $7.9-28.0$ & $2620-88830$ \\
\hline \\
a Minimum values of the conversion losses and noise temper- \\
atures and values at the available LO power are separated by \\
a "-".
\end{tabular}

values $T_{\text {noise }, d}$ higher than for the other two mixers. This performance of $T_{\text {noise, } d}$ and the degradation of the conversion losses at high frequencies described in Fig. 15 lead to the increase of the minimum values of $T_{\text {mix,DSB }}$ when the operation frequency increases, see Fig. 16.

An in-depth analysis of the impact of the epilayer length and the epilayer doping on the performance of the $1.2 \mathrm{THz}$ SHP mixer, the $2.5 \mathrm{THz}$ fundamental mixer and the $4.75 \mathrm{THz}$ fundamental mixer described in Table I has been carried out using the MCHB tool. The optimum values of $N_{e}$ and $L_{e}$ for these mixers and the predicted conversion losses and noise temperatures are compiled in Table III. In the optimization of these mixers, the circuit impedances at the RF and the LO frequencies have been conjugately matched at each operating point. The output impedances at the IF frequencies have been fixed to the values provided in Table I. In addition, the bias voltage and the anode area shown in Table I have been used in the optimization. The results obtained using the mixer designs in Table I are included in Table III for comparison. The reduction of the conversion losses after optimization of both the device structure and the circuit (impedance matching) has direct impact on the noise temperature, as can be seen using (3) and (4).

\section{Frequency Mixers Performance Evaluated With DIFFERENT MODELS}

Fig. 17 shows a comparison of the conversion losses and the noise temperature of some mixers described in Table I, obtained with different models coupled to the multi-tone HB tool. Also, measured results available in the literature have been included. The following models have been used in this analysis: A lumped equivalent circuit based on the constant series resistance presented in Table I (denoted by $\mathrm{LEC}_{R_{s, \mathrm{dc}}} \mathrm{HB}$ ); a lumped equivalent circuit based on a time varying series resistance $\left(\mathrm{LEC}_{R_{s}(t)} \mathrm{HB}\right)$; drift-diffusion models based on the steady-state velocity-field curve of GaAs (DDHB) and the constant low field mobility of GaAs $\left(\mathrm{DD}_{0} \mathrm{HB}\right)$. The noise temperature of the mixers has been calculated with the MCHB tool as described in Subsection II.D and with LEC models using the conversion matrix (CM) formalism presented in [6] and [29], see Appendix A. The traditional formulation of the $\mathrm{CM}$ uses a $\mathrm{LEC}_{R_{s, \mathrm{dc}}}$ model and it takes into account shot and thermal noise contributions. The effects of the modulation of the shot noise source under the LO signal is taken into account by using analytical noise correlation matrices. Hot electron noise and the effects of its modulation by the LO have been also included in the $\mathrm{CM}$ as described in [56]. Additionally, when we use the $\mathrm{LEC}_{R_{s}(t)}$ model in the CM, we have also taken into account the effects of the modulation of the thermal noise by the LO signal [57].

The models described in previous paragraph and used in Fig. 17 are commonly used in mixer design [5], [15], [34], [47], [49]. In [51], a lumped equivalent circuit model that takes into account carrier inertia and displacement current in the undepleted epilayer has been used to design a $1.2 \mathrm{THz}$ SHP mixer. This model uses an average electron temperature to take into account hot electron noise, neglecting the correlation contributions of this noise component. On the other hand, a drift-diffusion diode model coupled to a time-domain circuit simulator has been used in [58] for the analysis of a $2.5 \mathrm{THz}$ fundamental mixer. In [59], an ad-hoc improvement of the DD model to extend its validity under flat band conditions has been used to carry out an analysis of Schottky mixers.

Due to the accuracy of the MC model, the predictions of the MCHB tool have been considered as a reference to evaluate the accuracy and range of validity of other models. The most relevant results from Fig. 17 as regards the conversion losses are as follows.

- According to MCHB, velocity saturation does not affect the performance of the mixers up to $1 \mathrm{THz}$, due to the high doping and bias voltages used. As a consequence, a good agreement is observed between the predictions of MCHB and $\mathrm{DD}_{0} \mathrm{HB}$ which assumes a constant low field mobility.

- The traditional DD model (DDHB) fails when the diodes are working in the vicinity of flat band conditions. Above flat band, high electric fields are generated close to the Schottky contact. MC simulations show that nonlocal transport is important in this region. Since DD is a local model which uses a field dependent mobility, it overestimates the series resistance of this high field region. Also, local modeling of the electron transport in the $\mathrm{n}^{+}-\mathrm{n}$ transition with the DD model contributes to overestimate the series resistance of the diode. As a consequence, DD 


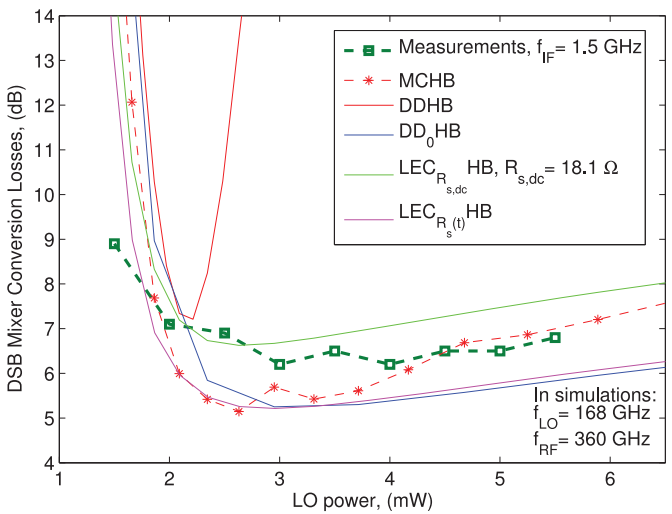

(a)

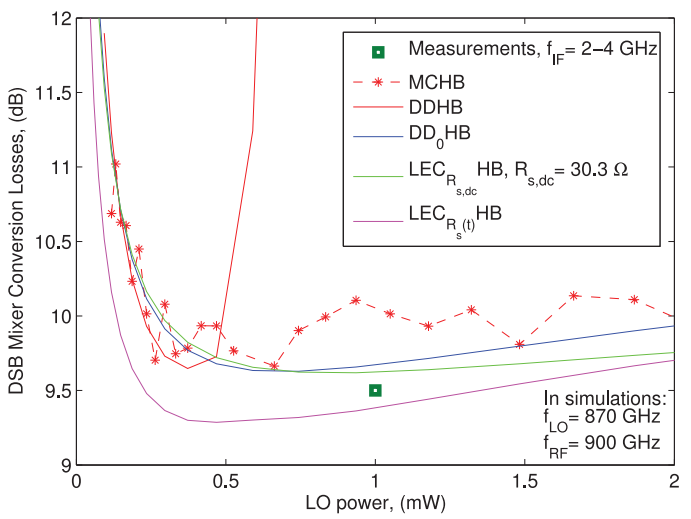

(c)

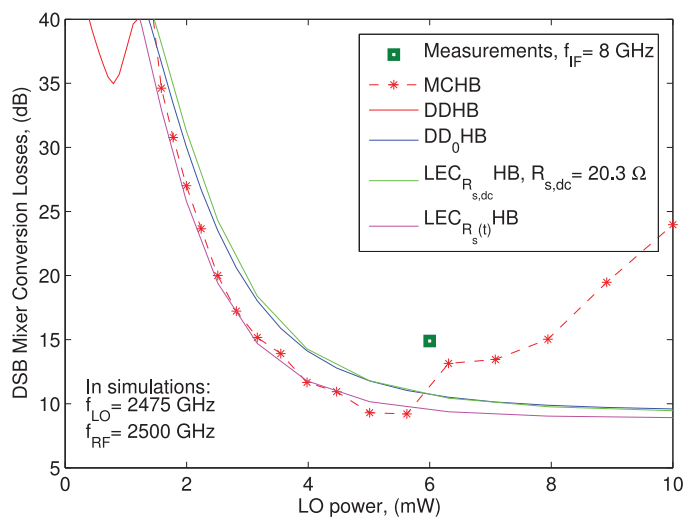

(e)

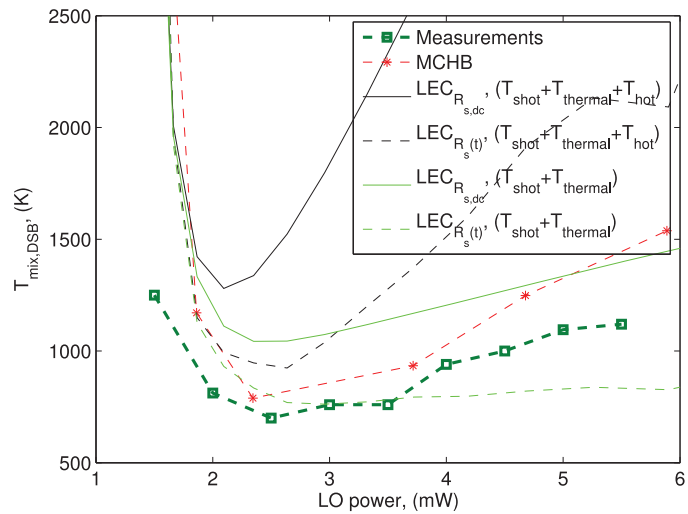

(b)

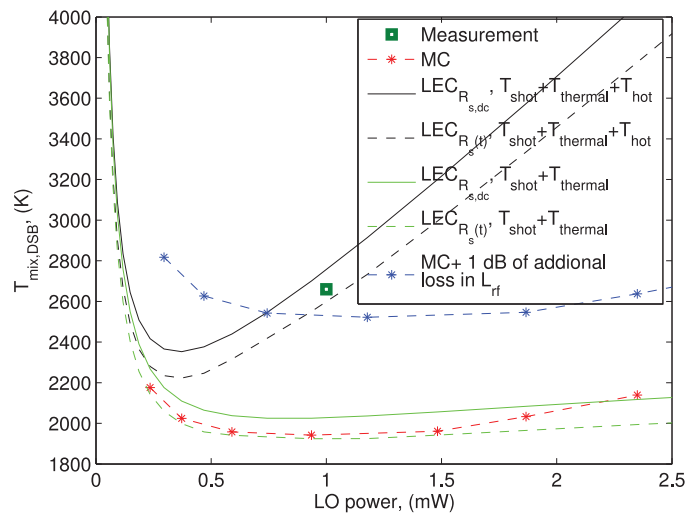

(d)

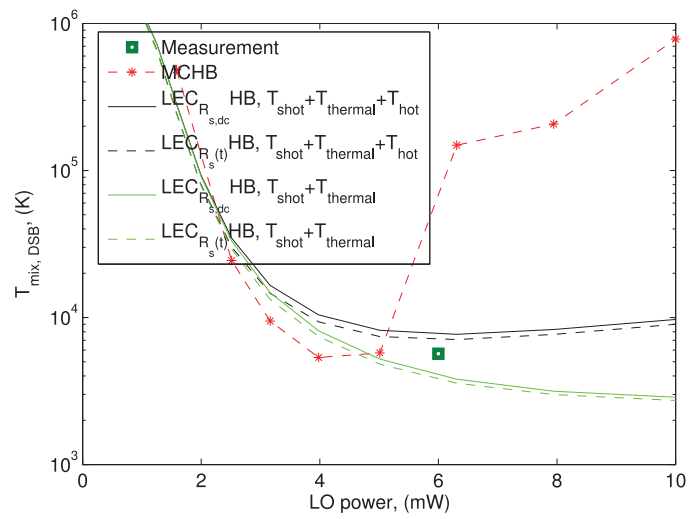

(f)

Fig. 17. DSB conversion losses (3) (left column) and equivalent input noise temperature (4) (right column) of the mixers described in Table I as a function of the LO power, simulated with LECHB, DDHB and MCHB tools. (a) 300-360 GHz subharmonic mixer, (b) 300-360 GHz subharmonic mixer, (c) 835-900 GHz fundamental balanced mixer, (d) $835-900 \mathrm{GHz}$ fundamental balanced mixer, (e) $2500 \mathrm{GHz}$ fundamental mixer, (f) $2500 \mathrm{GHz}$ fundamental mixer.

overestimates the conversion losses of the mixers. Since $\mathrm{DD}_{0}$ assumes a constant low field mobility independently of the electric field, it does not overestimate the series resistance, leading to a good agreement with the MC model.

- The predictions of the $\mathrm{LEC}_{R_{s}(t)} \mathrm{HB}$ are in good agreement with the results from $\mathrm{DD}_{0} \mathrm{HB}$. On the other hand, since LEC $_{R_{s}}$ HB uses a constant $R_{s}$, it overestimates the series resistance of the diode and, hence, overestimates the conversion losses.

- Velocity saturation is important in the performance of the $2.5 \mathrm{THz}$ mixer, see Section IV.A. $\mathrm{DD}_{0}$ and LEC models underestimate the conversion losses of this mixer, since they assume a constant low field electron mobility.
The most important conclusions from Fig. 17 as regards the noise temperature are:

- For the mixers up to $1 \mathrm{THz}$, the comparison of the results from the CM approach using different LEC models and MCHB indicates that the noise temperature at these frequencies is essentially determined by shot and thermal noise contributions. The calculation of the noise temperature with the CM approach is highly affected by the accuracy of the LEC model considered: Using a $\mathrm{LEC}_{R_{s}}$ model, the noise temperature is overestimated since this model overestimates the series resistance of the diode. Noise temperature evaluated with a time varying series resistance shows a better agreement with the results obtained with MCHB. 
- According to MCHB, hot electron noise contribution evaluated with the equations presented in [15], [60] is overestimated. The model in [15], [60] depends on the energy relaxation time $\tau_{\epsilon} . \tau_{\epsilon}$ is a function of the doping concentration and the electron energy, but it is used as an empirical parameter in the hot electron noise model. The results from MCHB provide a reference to analyze the limitations of this model.

- Results from Monte Carlo simulations indicate that intervalley transfer generates an important noise contribution for the $2.5 \mathrm{THz}$ mixer at $P_{\mathrm{LO}}$ higher than $4 \mathrm{~mW}$, see Subsection IV.A.

- Some discrepancies are observed between measurements and simulation results for the $835-900 \mathrm{GHz}$ fundamental balanced mixer, see Fig. 17(d). Since MC simulations indicate that there is not hot electron noise for this mixer, the observed discrepancies should be related to additional losses in other elements of the mixer. In fact, assuming additional losses of $1 \mathrm{~dB}$ in $L_{\mathrm{qo}}, L_{\mathrm{rf}}$ or $L_{\mathrm{if}}$, the observed discrepancies can be explained.

\section{CONCLUSION}

A reliable simulation tool for the analysis and design of Schottky-based mixers at millimeter and submillimeter wavelengths has been developed by combining a Monte Carlo device model with a multi-tone harmonic balance technique.

We have obtained good agreement between published experimental results and calculations with MCHB for both conversion losses and noise temperatures of Schottky mixers up to $2.5 \mathrm{THz}$. In addition, a significant reduction of the intervalley scattering noise which appears at high frequencies has been predicted by the MCHB tool when the epilayer length is decreased.

The analysis of Schottky mixers using different diode models has shown that $\mathrm{DD}_{0}$ and improved LEC models describe correctly the conversion losses when there is no velocity saturation (RF frequencies lower than $1 \mathrm{THz}$ ). On the other hand, the traditional DD model fails when the diodes are working close to flat band.

$\mathrm{Up}$ to frequencies around $1 \mathrm{THz}$, the noise temperature of mixers is usually well predicted by the ordinary shot and thermal noise theory. MC simulations have shown that common analytical models for hot electron noise integrated in the conversion matrix formalism overestimate this noise contribution. At frequencies higher than $1 \mathrm{THz}$, Monte Carlo simulations have shown an important increase of the noise temperature due to intervalley transfer.

\section{APPENDIX \\ CONVERSION MATRIX FORMALISM}

This appendix presents an overview of the conversion matrix (CM) formalism for the analysis of Schottky mixers [6], [29], [36]. The CM or large-signal-small-signal approach assumes that the nonlinear device is pumped by a single large sinusoidal signal (LO) together with another signal with much smaller amplitude (RF). The following steps summarize the procedure to calculate the conversion losses and the noise temperature of mixers.

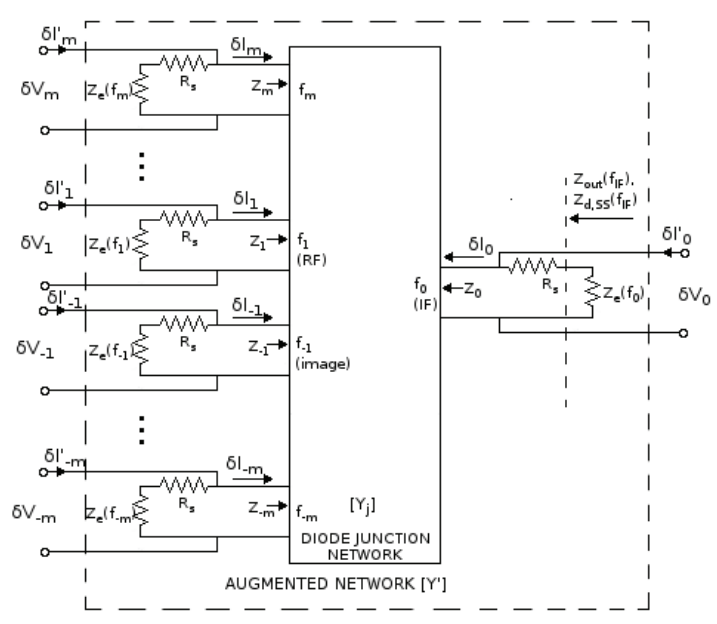

Fig. 18. Small signal representation of the mixer as a multifrequency linear multiport circuit. The voltage and current $\delta V_{m}$ and $\delta I_{m}^{\prime}$ at any port $m$ are the small-signal components at frequency $f_{\mathrm{IF}}+m f_{\mathrm{LO}}$ of the augmented network; each port represents one sideband frequency. During normal mixer operation the equivalent signal current generator is connected at port 1 of the augmented network, the other ports being open-circuited. In the noise analysis, equivalent noise current sources $\delta I_{S, m}^{\prime}, \delta I_{T, m}^{\prime}$ and $\delta I_{H, m}^{\prime}$ are connected to all ports. Reproduced from [6]

- Simulation of the nonlinear circuit under the large-signal LO excitation only, using the harmonic balance method together with a diode model. A lumped equivalent circuit model of the diode is commonly used in the CM formalism.

- The nonlinear elements of the diode modulated by the LO waveforms obtained from the first step are converted into linear, time varying elements, using the incremental conductance $g_{j}(t)$ and capacitance $c_{j}(t)$ of the Schottky junction.

- The small-signal voltages $v(t)$ and currents $i(t)$ at the terminals of the diode are analyzed in the frequency domain (the vectors of the phasor components of $v(t)$ and $i(t)$ at the mixing frequencies $f_{n}=f_{\mathrm{IF}}+n f_{\mathrm{LO}}$ where $n$ is an integer, are denoted by $\mathbf{V}$ and $\mathbf{I}$, respectively), see Fig. 18. $\mathbf{V}$ and $\mathbf{I}$ are related by means of a conversion matrix $\mathbf{Y}_{j}, \mathbf{I}=\mathbf{Y}_{j} \mathbf{V}=\left(\mathbf{G}_{j}+j \boldsymbol{\Omega} \mathbf{C}_{j}\right) \mathbf{V}$, see the notation used in [6] and [29].

- The impedance of the diode at the different mixing frequencies and the conversion losses of the mixer can be directly evaluated with the conversion matrix. At the IF frequency, the output impedance of the diode can be found by setting a signal source at the IF frequency, and calculating [29]

$$
Z_{\text {out }}\left(f_{\mathrm{IF}}\right)=\frac{V_{s}\left(f_{\mathrm{IF}}\right)}{I_{\mathrm{IF}}}-Z_{e}\left(f_{\mathrm{IF}}\right)
$$

where $V_{s}\left(f_{\mathrm{IF}}\right)$ is the small-signal voltage source at frequency $f_{\mathrm{IF}}, I_{\mathrm{IF}}$ is the current phasors at $f_{\mathrm{IF}}$ and $Z_{e}\left(f_{\mathrm{IF}}\right)$ is the impedance of the circuit at $f_{\mathrm{IF}} \cdot Z_{\mathrm{out}}\left(f_{\mathrm{IF}}\right)$ is equivalent to the definition of $Z_{d, \mathrm{SS}}\left(f_{\mathrm{IF}}\right)$ in (5). These impedances, $Z_{\text {out }}\left(f_{\mathrm{IF}}\right)$ and $Z_{d, \mathrm{SS}}\left(f_{\mathrm{IF}}\right)$, represent the small-signal impedance seen at the terminals of the diode in the mixer circuit at frequency $f_{\mathrm{IF}}$, see Fig. 18.

- Noise is a small-signal phenomenon. So, the conversion matrix formalism together with analytical models for shot, thermal and hot electron noise contributions are used to 


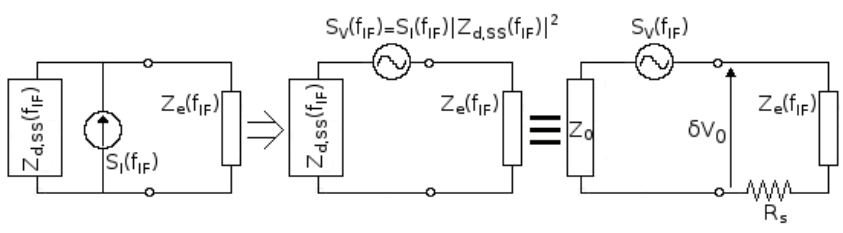

Fig. 19. The transformation of the current noise source at $f_{\mathrm{IF}}$ in parallel with the small-signal impedance $Z_{d, \mathrm{SS}}\left(f_{\mathrm{IF}}\right)$ to an equivalent voltage noise source in series with $Z_{d, \mathrm{SS}}\left(f_{\mathrm{IF}}\right)$ by means of Thévenin's theorem.

calculate the noise correlation matrices and evaluate the equivalent input noise temperature of the mixer. Let $\delta I_{m}^{\prime}$ denote the phasor of the noise current at each mixing frequency $f_{m}$, see Fig. 18. The noise correlation matrices of the different noise sources can be found in the literature [6], [15], [29].

- The noise power delivered to the output load at IF frequency is [29]

$$
P_{\text {del }}\left(f_{\mathrm{IF}}\right)=\frac{\left\langle\left|\delta V_{0}\right|^{2}\right\rangle \operatorname{Re}\left\{Z_{e}\left(f_{\mathrm{IF}}\right)\right\}}{\left|Z_{e}\left(f_{\mathrm{IF}}\right)+R_{s}\right|^{2}} .
$$

where $\left\langle\left|\delta V_{0}\right|^{2}\right\rangle$ is the total mean-squared noise voltage at $f_{\mathrm{IF}}$ [6], [29], [36]. This equation is equivalent to (7) used in the MC simulations. Using Thévenin's theorem in the circuit of Fig. 3, the equivalent circuit of the noisy diode at IF frequency can be represented by a voltage noise source characterized by $S_{V}\left(f_{\mathrm{IF}}\right)=S_{I}\left(f_{\mathrm{IF}}\right)\left|Z_{d, \mathrm{SS}}\left(f_{\mathrm{IF}}\right)\right|^{2}$ in series with $Z_{d, \mathrm{SS}}$, see Fig. 19. Using this transformation in (7), the noise power delivered to the output load $Z_{e}\left(f_{\mathrm{IF}}\right)$ is

$$
P_{\mathrm{del}}\left(f_{\mathrm{IF}}\right)=\frac{S_{V}\left(f_{\mathrm{IF}}\right) \Delta f \operatorname{Re}\left\{Z_{e}\left(f_{\mathrm{IF}}\right)\right\}}{\left|Z_{d, \mathrm{SS}}\left(f_{\mathrm{IF}}\right)+Z_{e}\left(f_{\mathrm{IF}}\right)\right|^{2}} .
$$

In the CM formalism with a LEC model of the diode, $Z_{\text {out }}\left(f_{\mathrm{IF}}\right)=Z_{0}+R_{s}$ where $Z_{0}$ is the impedance defined in Fig. 18 at IF frequency [6]. Equation (10) is expressed in terms of $\delta V_{0}$, i.e., the voltage fluctuations in the series combination of $R_{s}$ and $Z_{e}\left(f_{\mathrm{IF}}\right)$, see Fig. 18. In the circuit of Fig. 19, the small-signal impedance $Z_{d, \mathrm{SS}}\left(f_{\mathrm{IF}}\right)$ can be expressed as $Z_{d, \mathrm{SS}}\left(f_{\mathrm{IF}}\right) \equiv Z_{\text {out }}\left(f_{\mathrm{IF}}\right)=Z_{0}+R_{s}$ and the voltage noise spectral density can be written as $S_{V}\left(f_{\mathrm{IF}}\right) \Delta f$ $=\left\langle\left|\delta V_{\mathrm{ns}, 0}\right|^{2}\right\rangle$. According to Fig. $19, \delta V_{0}$ and $\delta V_{\mathrm{ns}, 0}$ are related by

$$
\begin{aligned}
\delta V_{\mathrm{ns}, 0} & =\frac{Z_{0}+R_{s}+Z_{e}\left(f_{\mathrm{IF}}\right)}{Z_{e}\left(f_{\mathrm{IF}}\right)+R_{s}} \delta V_{0} \\
& =\frac{Z_{d, \mathrm{SS}}\left(f_{\mathrm{IF}}\right)+Z_{e}\left(f_{\mathrm{IF}}\right)}{Z_{e}\left(f_{\mathrm{IF}}\right)+R_{s}} \delta V_{0} .
\end{aligned}
$$

$S_{V}\left(f_{\mathrm{IF}}\right) \Delta f=\left\langle\left|\delta V_{\mathrm{ns}, 0}\right|^{2}\right\rangle$ can be written in terms of $\delta V_{0}$ by using (12). When this expression of $S_{V}\left(f_{\mathrm{IF}}\right)$ is used in (11), we recover (10).

\section{REFERENCES}

[1] T. W. Crowe, W. L. Bishop, D. W. Porterfield, J. L. Hesler, I. Weikle, and R. M. , "Opening the terahertz window with integrated diode circuits," IEEE J. Solid-State Circuits, vol. 40, no. 10, pp. 2104-2110, Oct. 2005.

[2] P. Siegel, “THz instruments for space," IEEE Trans. Antennas Propag., vol. 55 , no. 11 , pp. $2957-2965$, Nov. 2007.
[3] G. Chattopadhyay, "Technology, capabilities, and performance of low power terahertz sources," IEEE Trans. THz Sci. Technol., vol. 1, no. 1, pp. 33-53, Sep. 2011.

[4] P. H. Siegel, "Terahertz technology," IEEE Trans. Microw. Theory Techn., vol. 50, no. 5, pp. 910-928, Mar. 2002.

[5] A. Maestrini et al., "Schottky diode-based terahertz frequency multipliers and mixers," C. R. Phys., vol. 11, no. 7-8, pp. 480-495, Aug. 2010.

[6] D. Held and A. Kerr, "Conversion loss and noise of microwave and millimeterwave mixers: Part I-Theory," IEEE Trans. Microw. Theory Techn., vol. MTT-26, no. 2, pp. 49-55, Feb. 1978.

[7] K. Champlin and G. Eisenstein, "Cutoff frequency of submillimeter Schottky-barrier diodes," IEEE Trans. Microw. Theory Techn., vol. MTT-26, no. 1, pp. 31-34, Jan. 1978.

[8] T. W. Crowe, "GaAs Schottky barrier mixer diodes for the frequency range 1-10 THz," Int. J. Infrared Millim. Waves, vol. 10, no. 7, pp. 765-777, Jul. 1989.

[9] E. Kolberg, T. Tolmunen, M. Frerking, and J. East, "Current saturation in submillimeter-wave varactors," IEEE Trans. Microw. Theory Techn., vol. 40, no. 5, pp. 831-838, May 1992.

[10] J. East, "Monte Carlo simulation of Schottky barrier mixers and varactors," in Proc. 6th Int. Symp. Space Terahertz Tech., Pasadena, California, Mar. 1995

[11] W. L. Schroeder and I. Wolff, "Monte-Carlo study of high-frequency, large-signal transport parameters for physics based device simulation," IEEE Trans. Electron Devices, vol. 42, no. 5, pp. 819-827, May 1995.

[12] R. Lipsey et al., "Monte Carlo harmonic-balance and drift-diffusion harmonic-balance analyses of 100-600 GHz Schottky barrier varactor frequency multipliers," IEEE Trans. Electron Devices, vol. 44, no. 11, pp. $1843-1850$, Nov. 1997.

[13] J. Grajal, V. Krozer, E. Gonzalez, F. Maldonado, and J. Gismero, "Modeling and design aspects of millimeter-wave and submillimeter-wave Schottky diode varactor frequency multipliers," IEEE Trans. Microw. Theory Techn., vol. 48, no. 4, pp. 700-711, Apr. 2000.

[14] H. Zirath, "High-frequency noise and current-voltage characteristics of mm-wave platinum n-n+ GaAs Schottky barrier diodes," J. Appl. Phys., vol. 60, no. 4, p. 1399, 1986.

[15] T. Crowe and R. Mattauch, "Analysis and optimization of millimeterand submillimeter-wavelength mixer diodes," IEEE Trans. Microw. Theory Techn., vol. MTT-35, no. 2, pp. 159-168, Feb. 1987.

[16] K. M. van Vliet, "Markov approach to density fluctuations due to transport and scattering. II applications," J. Math. Phys., vol. 12, no. 9, pp. 1998-2012, 1971.

[17] V. Gruzinskis, E. Starikov, P. Shiktorov, L. Reggiani, and L. Varani, "Hydrodynamic approach to noise spectra in unipolar semiconductor structures," Appl. Phys. Lett., vol. 64, no. 13, pp. 1662-1664, Mar. 1994.

[18] F. Bonani and G. Ghione, Noise in Semiconductor Devices: Modeling and Simulation. Berlin, Germany: Springer-Verlag, 2001.

[19] F. Bonani, S. Guerrieri, and G. Ghione, "Physics-based simulation techniques for small- and large-signal device noise analysis in RF applications," IEEE Trans. Electron Devices, vol. 50, no. 3, pp. 633-644, Mar. 2003.

[20] M. V. Fischetti and S. E. Laux, "Monte Carlo analysis of electron transport in small semiconductor devices including band-structure and space-charge effects," Phys. Rev. B, vol. 38, no. 14, pp. 9721-9745, Nov. 1988.

[21] C. Jacoboni and P. Lugli, The Monte Carlo Method for Semiconductor Device Simulation. New York, NY, USA: Springer-Verlag, 1989.

[22] P. Shiktorov, E. Starikov, V. Gruzhinskis, S. Pérez, T. González, L. Reggiani, L. Varani, and J. C. Vaissiere, "Upconversion of partition noise in semiconductors operating under periodic large-signal conditions," Phys. Rev. B, vol. 67, 2003.

[23] P. Shiktorov et al., "Monte Carlo simulation of Schottky diodes operating under terahertz cyclostationary conditions," IEEE Electron Device Lett., vol. 25, no. 1, pp. 1-3, Jan. 2004.

[24] S. Pérez et al., "Noise in Schottky-barrier diodes: From static to largesignal operation," in Proc. SPIE, 2004, vol. 5470.

[25] D. Pardo, "Analysis and design of multipliers and mixers via monte carlo modeling at THz bands" Ph.D. dissertation, Univ. Politécnica de Madrid, Madrid, Spain, Dec. 2014 [Online]. Available: http://oa.upm. es/33736/

[26] C. Predmore, A. Raisanen, N. Erickson, P. Goldsmith, and J. L. Marrero, "A broad-band, ultra-low-noise Schottky diode receiver from 80 to $115 \mathrm{GHz}$ diode," IEEE Trans. Microw. Theory Techn., vol. 32, no. 5, pp. 498-507, May 1984. 
[27] W. Y. Ali-Ahmad, W. Bishop, T. Crowe, and G. Rebeiz, "An 86-106 GHz quasi-integrated low noise Schottky receiver," IEEE Trans. Microw. Theory Techn., vol. 41, no. 4, pp. 558-564, Apr. 1993.

[28] B. Thomas, "Etude et realisation dúne tete de reception heterodyne en ondes submillimetriques pour letude des atmospheres et surfaces de planetes," Ph.D. dissertation, Laboratoire détude du Rayonnement et de la Matiere en Astrophysique, Observatoire de Paris, , Dec. 2004.

[29] S. Maas, Microwave Mixers, 2nd ed. Boston, MA: Artech House, 1993.

[30] M. Lundstrom, Fundamentals of Carrier Transport, 2nd ed. Cambridge, U.K.: Cambridge Univ. Press, 2000.

[31] T. González, D. Pardo, L. Reggiani, and L. Varani, "Microscopic analysis of electron noise in GaAs Schottky barrier diodes," J. Appl. Phys., vol. 82 , no. 5, pp. 2349-2358, 1997.

[32] J.-P. Nougier, "Fluctuations and noise of hot carriers in semiconductor materials and devices," IEEE Trans. Electron Devices, vol. 41, no. 11, pp. 2034-2049, 1994

[33] D. Pardo, J. Grajal, S. Pérez, B. Mencía, J. Mateos, and T. González, "Analysis of noise spectra in GaAs and GaN Schottky barrier diodes," Semicond. Sci. Technol., vol. 26, no. 5, p. 055023, 2011.

[34] J. Hesler et al., "Fixed-tuned submillimeter wavelength waveguide mixers using planar Schottky-barrier diodes," IEEE Trans. Microw. Theory Techn., vol. 45, no. 5, pp. 653-658, May 1997.

[35] K. Kundert, G. Sorkin, and A. Sangiovanni-Vincentelli, "Applying harmonic balance to almost-periodic circuits," IEEE Trans. Microw. Theory Techn., vol. 36, no. 2, pp. 366-378, Feb. 1988.

[36] S. Maas, Nonlinear Microwave Circuits. Reading, MA, USA: Artech House, 1988, Incorporated.

[37] P. J. C. Rodrigues, Computer-Aided Analysis of Nonlinear Microwave Circuits. Boston, MA, USA: Artech House, 1997.

[38] X.-D. Zhang, X.-N. Hong, and B.-X. Gao, "An accurate Fourier transform method for nonlinear circuits analysis with multi-tone driven," IEEE Trans. Circuits Syst., vol. 37, no. 5, pp. 668-669, May 1990

[39] A. Van der Ziel, Noise; Sources, Characterization, Measurement. Englewood Cliffs, NJ, USA: Prentice-Hall, 1970.

[40] W. Gardner, "Signal interception: A unifying theoretical framework for feature detection," IEEE Trans. Commun., vol. 36, no. 8, pp. 897-906, Aug. 1988.

[41] M. Trippe, G. Bosman, and A. van der Ziel, "Transit-time effects in the noise of Schottky-barrier diodes," IEEE Trans. Microw. Theory Techn., vol. MTT-34, no. 11, pp. 1183-1192, Nov. 1986.

[42] J. L. Hesler, "Planar Schottky diodes in submillimeter-wavelength waveguide receivers," Ph.D. dissertation, University of Virginia, Blacksburg, VA, USA, Jan. 1996.

[43] H. Torrey, C. Whitmer, and S. Goudsmit, Crystal Rectifiers. New York, NY, USA: McGraw-Hill, 1948.

[44] E. H. Rhoderick and R. H. Williams, Metal-Semiconductor Contacts, 2nd ed. Oxford, U.K.: Clarendon, 1988.

[45] C. Burckhardt, "Analysis of varactor frequency multipliers for arbitrary capacitance variation and drive level," Bell Syst. Tech. J., vol. 44, no. 4, pp. 675-692, Apr. 1965

[46] W. Ali-Ahmad, "Millimeter and submillimeter-wave integrated horn antenna Schottky receivers," Ph.D. dissertation, Univ. Michigan, CAMPUS???, 1993.

[47] B. Thomas, A. Maestrini, and G. Beaudin, "A low-noise fixed-tuned 300-360 GHz sub-harmonic mixer using planar Schottky diodes," IEEE Microw. Wireless Compon. Lett., vol. 15, no. 12, pp. 865-867, Dec. 2005.

[48] B. Thomas et al., "An $874 \mathrm{GHz}$ fundamental balanced mixer based on MMIC membrane planar Schottky diodes," in Proc. 21st Int. Symp. Space Terahertz Tech., Oxford, UK, Mar. 2010.

[49] B. Thomas et al., "A broadband $835-900 \mathrm{GHz}$ fundamental balanced mixer based on monolithic GaAs membrane Schottky diodes," IEEE Trans. Microw. Theory Techn., vol. 58, no. 7, pp. 1917-1924, Jul. 2010

[50] B. Thomas and J. Siles et al., " $560 \mathrm{GHz}, 664 \mathrm{GHz}$ and $1.2 \mathrm{THz}$ Schottky based MMIC sub-harmonic mixers for planetary atmospheric remote sensing and FMCW radar," in Proc. 22rd Int. Symp. Space THz Tech., Tucson, AZ, Apr. 2011, pp. 139-142.

[51] E. Schlecht et al., "Schottky diode based $1.2 \mathrm{THz}$ receivers operating at room-temperature and below for planetary atmospheric sounding," IEEE Trans. THz Sci. Technol., vol. 4, no. 6, pp. 661-669, Nov. 2014.

[52] P. H. Siegel, R. P. Smith, M. C. Graidis, and S. C. Martin, " $2.5 \mathrm{THz}$ GaAs monolithic membrane-diode mixer," IEEE Trans. Microw. Theory Techn., vol. 47, pp. 596-604, May 1999.
[53] A. Betz and R. Boreiko, "A practical Schottky mixer for $5 \mathrm{THz}$ (Part II)," in Proc. 7th Int. Symp. Space Terahertz Tech., Mar. 1996, p. 503.

[54] K. Hui, J. Hesler, D. Kurtz, W. Bishop, and T. Crowe, "A micromachined $585 \mathrm{GHz}$ Schottky mixer," IEEE Microw. Guided Wave Lett. vol. 10, no. 9, pp. 374-376, Sept. 2000.

[55] B. Thomas, A. Maestrini, D. Matheson, I. Mehdi, and P. de Maagt, "Design of an $874 \mathrm{GHz}$ biasable sub-harmonic mixer based on MMIC membrane planar Schottky diodes," in Proc. 33rd Int. Conf. Infrared, Millim., and THz Waves, Sep. 2008, pp. 1-2.

[56] T. Crowe, R. Mattauch, H. Roser, W. Bishop, W. Peatman, and X. Liu, "GaAs Schottky diodes for THz mixing applications," Proc. IEEE, vol. 80, no. 11, pp. 1827-1841, Nov. 1992.

[57] S. Maas, Noise in Linear and Nonlinear Circuits. : Artech House, 2005.

[58] H. Wang, S. Jones, G. Tait, and C. Mann, T. W. Crowe and R. M Weikle, Eds., "Combined circuit-device time domain simulation of 2.5 THz GaAs Schottky diode mixers," in Proc. 10th Int. Symp. Space THz Tech., Mar. 1999, p. 419.

[59] J. Siles, J. Grajal, and A. Di Carlo, "Design of submillimeter Schottky mixers under flat-band conditions using an improved drift-diffusion model," IEEE Microw. Wireless Compon. Lett., vol. 19, no. 3, pp. 167-169, Mar. 2009.

[60] G. Hegazi, A. Jelenski, and K. Yngvesson, "Limitations of microwaveand millimeter-wave mixers due to excess noise," IEEE Trans. Microw. Theory Techn., vol. 33, no. 12, pp. 1404-1409, Dec. 1985.

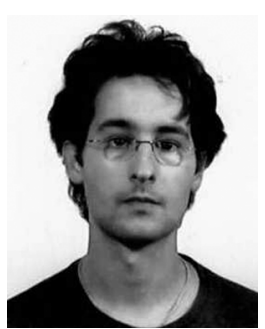

Diego Pardo was born in Salamanca, Spain, in 1984. He received the degree in theoretical physics from the Universidad de Salamanca, Salamanca, Spain, in 2008, and the Ph.D. degree from the Universidad Politécnica de Madrid, Madrid, Spain, in 2014.

Since 2009, he has been with the Signals, System, and Radiocommunications Department at Technical University of Madrid, Madrid, Spain. His research activities are in the area of semiconductor device modeling, including physic-based noise analysis

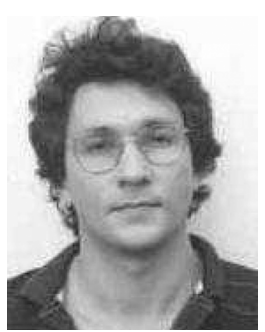

Jesús Grajal was born in Toral de los Guzmanes (León), Spain, in 1967. He received the Ingeniero de Telecomunicación degree and the Ph.D. degree from the Universidad Politécnica de Madrid, Madrid, Spain, in 1992 and 1998, respectively.

In 1993, he joined the Signal, System, and Radiocommunications Department at Technical University of Madrid, Madrid, Spain, where he has been an Associate Professor since 1998. His research activities are in the area of semiconductor device modeling and high-frequency circuit and system design.

Dr. Grajal was a co-recipient of the 2013 EuCAP best antenna design paper award.

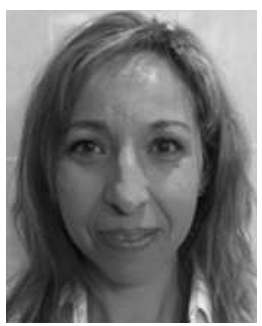

Susana Pérez was born in Salamanca, Spain, in 1970. She received the M.S. degree in physics and the Ph.D. degree in physics from the Universidad de Salamanca, Salamanca, in 1993 and 1999, respectively.

Since 1994, she has been with the Applied Physics Department, Universidad de Salamanca, Salamanca, Spain, where she became an Associate Professor in 2009. She was a Visiting Researcher with the LCR of Thomson-CSF (Thales), Orsay, France, and with the IRCOM (Xlim), Limoges, France. Her current research interests are in the development of terahertz devices and the field of numerical simulation of electronic transport and noise in submicrometer semiconductor devices. 